\title{
Study of a Class of Generalized Multiterm Fractional Differential Equations with Generalized Fractional Integral Boundary Conditions
}

\author{
Wafa Shammakh (D), Hadeel Z. Alzumi $(D$, and Zahra Albarqi \\ Mathematics Department, Faculty of Science, University of Jeddah, Jeddah, Saudi Arabia \\ Correspondence should be addressed to Hadeel Z. Alzumi; hzalzumi@uj.edu.sa
}

Received 31 December 2019; Accepted 25 April 2020; Published 11 May 2020

Guest Editor: Thabet Abdeljawad

Copyright (c) 2020 Wafa Shammakh et al. This is an open access article distributed under the Creative Commons Attribution License, which permits unrestricted use, distribution, and reproduction in any medium, provided the original work is properly cited.

\begin{abstract}
The aim of this work is to study the new generalized fractional differential equations involving generalized multiterms and equipped with multipoint boundary conditions. The nonlinear term is taken from Orlicz space. The existence and uniqueness results, with the help of contemporary tools of fixed point theory, are investigated. The Ulam stability of our problem is also presented. The obtained results are well illustrated by examples.
\end{abstract}

\section{Introduction}

Fractional differential equations (FDEs) have a considerable interest both in mathematics and in applications. They were used in modeling in various disciplines of engineering, physics, chemical technology, population dynamics, biotechnology, economics, etc. (see, for example, [1-10], and the references cited therein). Recently, some authors explored FDEs with various types of conditions as multipoint, nonlocal, antiperiodic, and integral boundary conditions [11-15].

Furthermore, there has been a significant development in fractional derivatives and integrals due to the necessity of a better model for real phenomena. In [16], Katugampola suggested a new fractional integral that combines the Riemann-Liouville and Hadamard integrals into a single integral. In 2015, Caputo and Fabrizio [17] defined the socalled Caputo-Fabrizio fractional derivatives by imposing a nonsingular exponential kernel. Later, the exponential kernel was replaced by the Mittag-Leffler function [18, 19]. Another definition of generalized proportional fractional derivatives-generated Caputo and Riemann-Liouville involving exponential functions in their kernels was introduced by Jarad et al. [20]. Another new type of new fractional derivative can be found in [21]. An application on such derivatives can be seen in [22]. More recently, generalized fractional derivatives that contain kernels depending on the function on the space of absolutely continuous functions were investigated [23]. Following the above points, many authors established various generalized fractional boundary value problems (GFBVPs) (see, for example, [24-27]). It is worth to mention that some authors worked on the existence of solutions and studied the stability analysis for nonlinear singular fractional differential equations. For more details, we refer to [28-30].

On the other hand, Birnbaum and Orlicz [31] proposed a more general setting of function spaces in 1931, which were called Orlicz spaces. These spaces are the generalization of the classical Lebesgue spaces $L^{p}(1<p<\infty)$, where the kernel is given by a convex function instead of $x^{p}$. Some results that deal with differential equations in the framework of Orlicz spaces can be found in $[32,33]$.

In this paper, we investigate the boundary value problem as follows: 


$$
\left\{\begin{array}{l}
{ }^{C} D_{0^{+}}^{\alpha, \rho} u(t)=f\left(t, u(t),{ }^{C} D_{0^{+}}^{\alpha-1, \rho} u(t),{ }^{C} D_{0^{+}}^{\alpha-2, \rho} u(t),{ }^{C} D_{0^{+}}^{\alpha-3, \rho} u(t)\right), \quad t \in[0,1], \rho>0, \\
u(0)=\delta u(0)=\delta^{2} u(0)=0, \\
\int_{0}^{1} u(s) \mathrm{d} s=\sum_{i=1}^{m} \delta_{i} I^{\beta, \rho} u\left(\xi_{i}\right), \quad 0<\xi_{i}<1, \delta=t^{1-\rho} \frac{\mathrm{d}}{\mathrm{d} t},
\end{array}\right.
$$

where ${ }^{C} D_{0^{+}}^{\alpha, \rho}$ is the generalized fractional derivative of Caputo type of order $\alpha, 3<\alpha \leq 4, I_{0}^{\beta, \rho}$ denotes the Katugampola-type fractional integral of order $0<\beta<1, f$ is defined on an Orlicz space $L_{F}([0,1])$, and $\delta_{i} \in \mathbb{R},(i=$ $1, \ldots, m)$.

The rest of the paper is organized as follows. In Section 2, we will briefly recall some preliminary materials related to our problem. In Section 3, we discuss the existence and uniqueness results of GFBVP using some fixed point theorems and support the obtained results by using examples to well illustrate. The Ulam stability of our problem is given in Section 4.

\section{Preliminaries}

For convenience of the reader, we present some basic definitions about generalized fractional calculus theory, which can be found in $[16,34,35]$. Also, we introduce some necessary concepts for Orlicz spaces which are used throughout this paper. For more details about Orlicz spaces, one can see $[36,37]$.

Definition 1. Let $Q:[0, \infty) \longrightarrow[0, \infty)$ be a right continuous, monotone, increasing function with the following:
(i) $Q(0)=0$
(ii) $\lim _{t \rightarrow \infty} Q(t)=\infty$
(iii) $Q(t)>0$ whenever $t>0$

Then, the function defined by

$$
F(x)=\int_{0}^{x} Q(t) \mathrm{d} t, \quad x \geq 0,
$$

is called $N$-function. Alternatively, the function $F$ is an $N$-function iff $F$ is continuous, even, and convex with the following:
(i) $\lim _{x \longrightarrow 0}(F(x) / x)=0$
(ii) $\lim _{x \longrightarrow 0}(F(x) / x)=\infty$
(iii) $F(x)>0$ if $x>0$

Definition 2. For an $N$-function, we define

$$
F^{*}(x)=\int_{0}^{x} Q^{-1}(t) \mathrm{d} t, \quad x \geq 0,
$$

where $Q^{-1}$ is the right inverse of the right derivative of $F$ and is called the complementary of $F$ and it satisfies the following condition:

$$
F^{*}(x)=\sup \{t x-F(t): t \geq 0\}, \quad \forall x \geq 0 .
$$

Remark 1. Note that the function $F^{*}$ is also $N$-function and the complementary pairs $F$ and $F^{*}$ satisfy the following Young inequality:

$$
x t \leq F(x)+F^{*}(t), \quad \forall x, t \geq 0 .
$$

Definition 3. A function $F:[0, \infty) \longrightarrow[0, \infty)$ is called a Young function if it is convex and satisfies the conditions $F(0)=\lim _{x \longrightarrow 0^{+}} F(x)=0$ and $\lim _{x \rightarrow \infty} F(x)=\infty$.

Definition 4 (Orlicz space). For an $N$-function $F$, the Orlicz space $L_{F}([0,1])$ is the space of measurable functions $u:[0,1] \longrightarrow \mathbb{R}$ such that $\int_{0}^{1} F(|u(x)|) \mathrm{d} x<\infty$. This space endowed with the Luxemburg norm, i.e.,

$$
\|u\|_{F}=\inf \left\{\lambda>0: \int_{0}^{1} F\left(\frac{|u(x)|}{\lambda}\right) \mathrm{d} x \leq 1\right\},
$$

and the pair $\left(L_{F}([0,1]),\|u\|_{F}\right)$ is a Banach space.

Remark 2. For an Orlicz space, the Hölder inequality holds, that is,

$$
\int_{0}^{1} u v \mathrm{~d} x \leq 2\|u\|_{F}\|v\|_{F^{*}}
$$

where $u \in L_{F}([0,1])$ and $v \in L_{F^{*}}([0,1])$.

For generalized fractional calculus, Katugampola $[16,34,38]$ introduced the following definitions.

Definition 5 (Katugampola generalized fractional integral). The generalized fractional integral of order $\alpha \in \mathbb{C}$ with $\mathfrak{R}(\alpha)>0$ and $\rho>0$ for $-\infty<a<t<\infty$ is defined by

$$
\left(I_{a^{+}}^{\alpha, \rho} u\right)(t)=\frac{\rho^{1-\alpha}}{\Gamma(\alpha)} \int_{a}^{t} \frac{s^{\rho-1}}{\left(t^{\rho}-s^{\rho}\right)^{1-\alpha}} u(s) \mathrm{d} s,
$$

where $u$ belongs to the space $X_{c}^{p}(a, b)$, which denotes the space of all complex-valued Lebesgue measurable functions $f$ on $[a, b]$ for which $\|f\|_{X_{c}^{p}}<\infty$, where the norm is defined by

$$
\|f\|_{X_{c}^{p}}=\left(\int_{a}^{b}\left|t^{c} f(t)\right|^{p} \frac{\mathrm{d} t}{t}\right)^{1 / p}<\infty, \quad 1 \leq p<\infty, c \in \mathbb{R} .
$$

Note that integral (8) is called the left-sided generalized fractional integral. 
Definition 6 (Katugampola generalized Caputo fractional derivative). The left generalized fractional derivative of order $\alpha \in \mathbb{C}$ with $\mathfrak{R}(\alpha)>0$ and $\rho>0$ of $u \in A C_{\delta}^{n}[a, b]$, where $-\infty<a<t<\infty$, is defined by

$$
\begin{aligned}
\left({ }^{C} D_{a^{+}}^{\alpha, \rho} u\right)(t) & =\frac{1}{\Gamma(n-\alpha)} \int_{a}^{t}\left(\frac{t^{\rho}-s^{\rho}}{\rho}\right)^{n-\alpha-1} \frac{\left(\delta^{n} u\right)(s) \mathrm{d} s}{s^{1-\rho}} \\
& =I_{a^{+}}^{n-\alpha, \rho}\left(\delta^{n} u\right)(s)
\end{aligned}
$$

where $n=[\alpha]+1$ and $A C_{\delta}^{n}[a, b]$ denotes the space of all absolutely continuous real valued functions on $[a, b]$, where

$$
\begin{aligned}
& A C_{\delta}^{n}[a, b]=\left\{f:[a, b] \longrightarrow \mathbb{C} \text { and } \delta^{n-1} f \in A C[a, b], \delta=t^{1-\rho} \frac{\mathrm{d}}{\mathrm{d} t}\right\}, \\
& C_{\delta, \epsilon}^{n}[a, b]=\left\{f:[a, b] \longrightarrow \mathbb{C} \text { and } \delta^{n-1} f \in C[a, b], \delta^{n} f \in C_{\epsilon, \rho}[a, b], \delta=t^{1-\rho} \frac{\mathrm{d}}{\mathrm{d} t}\right\},
\end{aligned}
$$

for $0 \leq \epsilon \leq 1$, endowed with the norms $\|f\|_{C_{s}^{n}}=\sum_{k=0}^{n}\left\|\delta^{k} f\right\|_{C}$ and $\|f\|_{C^{n}}=\sum_{k=0}^{n-1}\left\|\delta^{k} f\right\|_{C}+\left\|\delta^{n} f\right\|_{C_{\epsilon, \rho}}$, respectively.

Next, we recall some basic properties of generalized fractional integral and derivative [39].

Lemma 1. Let $\mathfrak{R}(\alpha) \geq 0, n=[\Re(\alpha)+1]$, and $f \in A C_{\delta}^{n}[a$, $b]$, with $0<a<b<\infty$. For $k, m \in \mathbb{N}$,

$$
\begin{aligned}
& \left({ }_{a} I^{\alpha, \rho}\right)^{k}\left({ }_{a}^{C} D^{\alpha, \rho}\right)^{m} f(t) \\
& =\frac{\left({ }_{a}^{C} D^{\alpha, \rho}\right)^{m} f(\tau)}{\Gamma(k \alpha+1)}\left(\frac{t^{\rho}-a^{\rho}}{\rho}\right)^{k \alpha}, \quad \tau \in(a, t) .
\end{aligned}
$$

Theorem 1. Let $\mathfrak{R}(\alpha) \geq 0, n=[\Re(\alpha)+1]$, and $f \in A C_{\delta}^{n}[a$, $b]$, where $0<a<b<\infty$. Then,
(i) If $\alpha \notin \mathbb{N}_{0},\left({ }_{a}^{C} D^{\alpha, \rho} f\right)(x)={ }_{a} I^{n-\alpha, \rho}\left(\delta^{n} f\right)(x)$
(ii) If $\alpha \in \mathbb{N},\left({ }_{a}^{C} D^{\alpha, \rho} f\right)(x)=\left(\delta^{n} f\right)(x)$
(iii) ${ }_{a}^{C} D^{0, \rho} f=f$

Theorem 2. Let $f(x) \in C_{\delta}^{n}[a, b], 0<a<b<\infty$, and $\alpha, \beta \in \mathbb{C}$ such that $\mathfrak{R}(\alpha) \geq 0$ and $\mathfrak{R}(\beta) \geq 0$. Then,

$$
{ }_{a}^{C} D^{\alpha, \rho}{ }_{a} I^{\beta, \rho} f(x)={ }_{a} I^{\beta-\alpha, \rho} f(x) .
$$

Our main results are based on utilizing the following fixed point theorems.

Theorem 3 (Schaefer's fixed point theorem [40]). Let X be a Banach space. Assume that $\mathscr{L}: X \longrightarrow X$ is a completely continuous operator and the set $T=\{u \in X: u=$ $\lambda \mathscr{L} u, 0<\lambda<1\}$ is bounded. Then, $\mathscr{L}$ has a fixed point in $X$.

Theorem 4 (Krasnoselskii's fixed point theorem [40]). Let $\mathcal{N}$ be a closed, convex, bounded, and nonempty subset of a Banach space $X$. Let $T_{1}$ and $T_{2}$ be operators such that

(i) $T_{1}\left(u_{1}\right)+T_{2}\left(u_{2}\right)$ belong to $\mathcal{N}$ whenever $u_{1}, u_{2} \in \mathcal{N}$ (ii) $T_{1}$ is a compact and continuous and $T_{2}$ is a contraction mapping

Then, there exists $u_{0} \in \mathcal{N}$ such that $u_{0}=T_{1}\left(u_{0}\right)+T_{2}\left(u_{0}\right)$.

For computational convenience, we introduce the following notations:

$$
\psi=\frac{1}{\rho^{3}(3 \rho+1)}-\sum_{i=1}^{m} \delta_{i} \frac{\xi_{i}^{\rho(\beta+3)} \Gamma(4)}{\rho^{\beta+3} \Gamma(\beta+4)} .
$$

Lemma 2. Let $h \in L_{F}([0,1])$ and $\psi$ be given by (15). Then, the solution of the GFBVP

$$
\left\{\begin{array}{l}
{ }^{C} D_{0^{+}}^{\alpha, \rho} u(t)=h(t), t \in[0,1], \quad \rho>0, \\
u(0)=\delta u(0)=\delta^{2} u(0)=0, \\
\int_{0}^{1} u(s) \mathrm{d} s=\sum_{i=1}^{m} \delta_{i} I^{\beta, \rho} u\left(\xi_{i}\right), \quad \xi_{i} \in(0,1), \delta=t^{1-\rho} \frac{\mathrm{d}}{\mathrm{d} t},
\end{array}\right.
$$

is given by

$$
u(t)=I^{\alpha, \rho} h(t)+\frac{t^{3 \rho}}{\rho^{3} \psi}\left[\sum_{i=1}^{m} \delta_{i} I^{\alpha+\beta, \rho} h\left(\xi_{i}\right)-\int_{0}^{1} I^{\alpha, \rho} h(s) \mathrm{d} s\right] .
$$

Proof. By using Lemma 1, we obtain

$$
u(t)=I^{\alpha, \rho} h(t)+C_{0}+C_{1} \frac{t^{\rho}}{\rho}+C_{2} \frac{t^{2 \rho}}{\rho^{2}}+C_{3} \frac{t^{3 \rho}}{\rho^{3}},
$$

where $C_{i} \in \mathbb{R},(i=0,1,2,3)$ are the arbitrary constants. Using the boundary conditions $u(0)=\delta u(0)=\delta^{2} u(0)=0$, we get $C_{0}=C_{1}=C_{2}=0$. Then, from $\int_{0}^{1} u(s) \mathrm{d} s=\sum_{i=1}^{m}$ $\delta_{i} I^{\beta, \rho} u\left(\xi_{i}\right)$, it follows that 


$$
\begin{aligned}
C_{3}= & \frac{1}{\psi}\left[\sum_{i=1}^{m} \delta_{i} \frac{\rho^{1-(\alpha+\beta)}}{\Gamma(\alpha+\beta)} \int_{0}^{\xi_{i}}\left(\xi_{i}^{\rho}-s^{\rho}\right)^{\alpha+\beta-1} s^{\rho-1} h(s) \mathrm{d} s\right. \\
& \left.-\frac{\rho^{1-\alpha}}{\Gamma(\alpha)} \int_{0}^{1}\left(\int_{0}^{s}\left(s^{\rho}-\tau^{\rho}\right)^{\alpha} \tau^{\rho-1} h(\tau) \mathrm{d} \tau\right) \mathrm{d} s\right] .
\end{aligned}
$$

Substituting the values of $C_{0}, C_{1}, C_{2}$, and $C_{3}$ in (18), we get (17).

\section{Main Results}

In this section, we prove the existence and uniqueness of solutions of GFBVP (1). We assume that $f$ belongs to an
Orlicz space $L_{F}[0,1]$. For $3<\alpha \leq 4$, let $X=\left\{u: u,{ }^{C} D_{0^{+}}^{\alpha-1, \rho} u\right.$, $\left.{ }^{C} D_{0^{+}}^{\alpha-2, \rho} u,{ }^{C} D_{0^{+}}^{\alpha-3, \rho} u \in C([0,1], \mathbb{R})\right\}$ denote the Banach space of all continuous functions defined on $[0,1]$ into $\mathbb{R}$ endowed with the norm

$$
\begin{aligned}
\|u\|= & \sup \left\{|u(t)|+\left|{ }^{C} D_{0^{+}}^{\alpha-1, \rho} u(t)\right|+\left|{ }^{C} D_{0^{+}}^{\alpha-2, \rho} u(t)\right|\right. \\
& \left.+\left|{ }^{C} D_{0^{+}}^{\alpha-3, \rho} u(t)\right|, \quad t \in[0,1]\right\} .
\end{aligned}
$$

Relative to GFBVP (1), in view of Lemma 2, we define an operator $\mathscr{L}: X \longrightarrow X$ as

$$
\begin{aligned}
\mathscr{L} u(t)= & \frac{\rho^{1-\alpha}}{\Gamma(\alpha)} \int_{0}^{t}\left(t^{\rho}-s^{\rho}\right){ }^{\alpha-1} s^{\rho-1} f\left(s, u(s),{ }^{C} D_{0^{+}}^{\alpha-1, \rho} u(s),{ }^{C} D_{0^{+}}^{\alpha-2, \rho} u(s),{ }^{C} D_{0^{+}}^{\alpha-3, \rho} u(s)\right) \mathrm{d} s \\
& +\frac{t^{3 \rho}}{\rho^{3} \psi}\left[\sum_{i=1}^{m} \delta_{i} \frac{\rho^{1-(\alpha+\beta)}}{\Gamma(\alpha+\beta)} \int_{0}^{\xi_{i}}\left(\xi_{i}^{\rho}-s^{\rho}\right)^{\alpha+\beta-1} s^{\rho-1} f\left(s, u(s),{ }^{C} D_{0^{+}}^{\alpha-1, \rho} u(s),{ }^{C} D_{0^{+}}^{\alpha-2, \rho} u(s),{ }^{C} D_{0^{+}}^{\alpha-3, \rho} u(s)\right) \mathrm{d} s\right. \\
& \left.-\int_{0}^{1} I^{\alpha, \rho} f\left(s, u(s),{ }^{C} D_{0^{+}}^{\alpha-1, \rho} u(s),{ }^{C} D_{0^{+}}^{\alpha-2, \rho} u(s),{ }^{C} D_{0^{+}}^{\alpha-3, \rho} u(s)\right) \mathrm{d} s\right], \quad t \in[0,1] .
\end{aligned}
$$

Notice that GFBVP (1) has solutions if and only if the operator $\mathscr{L}$ has fixed points.

The following result plays a major role in our analysis.

Lemma 3. Let $F$ be a Young function which has a Young complement $F^{*}$ satisfying

$$
\begin{array}{r}
\int_{0}^{t} F^{*}\left(s^{\alpha-1}\right) \mathrm{d} s<\infty, \\
\int_{0}^{t} F^{*}\left(s^{\alpha+\beta-1}\right) \mathrm{d} s<\infty, \\
t>0,
\end{array}
$$

for $\alpha \in(3,4]$ and $\beta \in(0,1)$. Then, the operator $\mathscr{L}$ exists and is well defined.

Proof. Let $u \in X$. Define a function as follows:

$$
\varrho_{1}(s)= \begin{cases}\left(t^{\rho}-s^{\rho}\right)^{\alpha-1} s^{\rho-1}, & \text { if } s \in[0, t], t>0, \\ 0, & \text { otherwise. }\end{cases}
$$

At the beginning, we will show that $\varrho_{1} \in L_{F^{*}}[0,1]$. By using appropriate substitution and properties of the Young functions, one obtains

$$
\begin{aligned}
\int_{0}^{1} F^{*}\left(\frac{\left|\varrho_{1}(s)\right|}{\kappa}\right) \mathrm{d} s & =\int_{0}^{t} F^{*}\left(\frac{\left(t^{\rho}-s^{\rho}\right)^{\alpha-1}}{\kappa}\right) s^{\rho-1} \mathrm{~d} s \\
& =\frac{1}{\rho}\left(\frac{1}{\kappa}\right)^{1 /(1-\alpha)} \int_{0}^{\kappa^{1 /(1-\alpha)} t^{\rho}} F^{*}\left(s^{\alpha-1}\right) \mathrm{d} s
\end{aligned}
$$

by the assumption of the theorem, and we get $\varrho_{1} \in L_{F^{*}}[0,1]$. Similarly, setting

$$
\varrho_{2}(s)= \begin{cases}\left(t^{\rho}-s^{\rho}\right)^{\alpha+\beta-1} s^{\rho-1}, & \text { if } s \in[0, t], t>0 \\ 0, & \text { otherwise }\end{cases}
$$

one can get $\varrho_{2} \in L_{F^{*}}[0,1]$. Next, we show that $\mathscr{L}$ is well defined, i.e., $\mathscr{L} u(t) \in C([0,1], \mathbb{R})$. Let $0 \leq \tau<t \leq 1$. Thus, we have 


$$
\begin{aligned}
& |(\mathscr{L} u)(t)-(\mathscr{L} u)(\tau)| \leq \frac{\rho^{1-\alpha}}{\Gamma(\alpha)}\left[\int_{0}^{\tau}\left|\left[\left(t^{\rho}-s^{\rho}\right)^{\alpha-1}-\left(\tau^{\rho}-s^{\rho}\right)^{\alpha-1}\right] s^{\rho-1}\right|\right. \\
& \cdot\left|f\left(s, u(s),{ }^{C} D_{0^{+}}^{\alpha-1, \rho} u(s),{ }^{C} D_{0^{+}}^{\alpha-2, \rho} u(s),{ }^{C} D_{0^{+}}^{\alpha-3, \rho} u(s)\right)\right| \mathrm{d} s \\
& \left.+\int_{\tau}^{t} \mid\left(t^{\rho}-s^{\rho}\right)^{\alpha-1} s^{\rho-1}\left\|f\left(s, u(s),{ }^{C} D_{0^{+}}^{\alpha-1, \rho} u(s),{ }^{C} D_{0^{+}}^{\alpha-2, \rho} u(s),{ }^{C} D_{0^{+}}^{\alpha-3, \rho} u(s)\right)\right\| \mathrm{d} s\right] \\
& +\frac{\left(t^{3 \rho}-\tau^{3 \rho}\right)}{\rho^{3}|\psi|}\left[\sum_{i=1}^{m} \delta_{i} \frac{\rho^{1-(\alpha+\beta)}}{\Gamma(\alpha+\beta)} \int_{0}^{\xi_{i}}\left|\left(\xi_{i}^{\rho}-s^{\rho}\right)^{\alpha+\beta-1} s^{\rho-1}\right|\right. \\
& \cdot\left|f\left(s, u(s),{ }^{C} D_{0^{+}}^{\alpha-1, \rho} u(s),{ }^{C} D_{0^{+}}^{\alpha-2, \rho} u(s),{ }^{C} D_{0^{+}}^{\alpha-3, \rho} u(s)\right)\right| \mathrm{d} s \\
& -\frac{\rho^{1-\alpha}}{\Gamma(\alpha)} \int_{0}^{1}\left(\int_{0}^{s}\left|\left(s^{\rho}-\tau^{\rho}\right)^{\alpha-1} \tau^{\rho-1}\right|\right. \\
& \left.\left.\cdot\left|f\left(\tau, u(\tau),{ }^{C} D_{0^{+}}^{\alpha-1, \rho} u(\tau),{ }^{C} D_{0^{+}}^{\alpha-2, \rho} u(\tau),{ }^{C} D_{0^{+}}^{\alpha-3, \rho} u(\tau)\right)\right| \mathrm{d} \tau\right) \mathrm{d} s\right] \\
& \leq \frac{\rho^{1-\alpha}}{\Gamma(\alpha)}\left[\int_{0}^{1}\left[\mathscr{G}_{1}(s)+\mathscr{G}_{2}(s)\right]\left|f\left(s, u(s),{ }^{C} D_{0^{+}}^{\alpha-1, \rho} u(s){ }^{C} D_{0^{+}}^{\alpha-2, \rho} u(s),{ }^{C} D_{0^{+}}^{\alpha-3, \rho} u(s)\right)\right| \mathrm{d} s\right. \\
& +\frac{\left(t^{3 \rho}-\tau^{3 \rho}\right)}{\rho^{3}|\psi|}\left[\sum_{i=1}^{m} \delta_{i} \frac{\rho^{1-(\alpha+\beta)}}{\Gamma(\alpha+\beta)} \int_{0}^{\xi_{i}}\left|\left(\xi_{i}^{\rho}-s^{\rho}\right)^{\alpha+\beta-1} s^{\rho-1}\right|\right. \\
& \cdot\left|f\left(s, u(s),{ }^{C} D_{0^{+}}^{\alpha-1, \rho} u(s),{ }^{C} D_{0^{+}}^{\alpha-2, \rho} u(s),{ }^{C} D_{0^{+}}^{\alpha-3, \rho} u(s)\right)\right| \mathrm{d} s \\
& -\frac{\rho^{1-\alpha}}{\Gamma(\alpha)} \int_{0}^{1}\left(\int_{0}^{s}\left|\left(s^{\rho}-\tau^{\rho}\right)^{\alpha-1} \tau^{\rho-1}\right|\right. \\
& \left.\left.\cdot\left|f\left(\tau, u(\tau),{ }^{C} D_{0^{+}}^{\alpha-1, \rho} u(\tau),{ }^{C} D_{0^{+}}^{\alpha-2, \rho} u(\tau){ }^{C} D_{0^{+}}^{\alpha-3, \rho} u(\tau)\right)\right| \mathrm{d} \tau\right) \mathrm{d} s\right],
\end{aligned}
$$

where

$$
\mathscr{G}_{1}(s)= \begin{cases}\left|\left[\left(t^{\rho}-s^{\rho}\right)^{\alpha-1}-\left(\tau^{\rho}-s^{\rho}\right)^{\alpha-1}\right] s^{\rho-1}\right|, & \text { if } s \in[0, \tau] \\ 0, & \text { otherwise }\end{cases}
$$

$\mathscr{G}_{2}(s)= \begin{cases}\left|\left(t^{\rho}-s^{\rho}\right)^{\alpha-1} s^{\rho-1}\right|, & \text { if } s \in[\tau, t] \\ 0, & \text { otherwise. }\end{cases}$
The functions $\mathscr{G}_{i}, i=1,2$, belong to $L_{F^{*}}[0,1]$ with $\left\|\mathscr{G}_{i}\right\|_{F^{*}} \leq g\left(\left|t^{\rho}-\tau^{\rho}\right|\right), i=1,2$, where $g: \mathbb{R}^{+} \longrightarrow \mathbb{R}^{+}$is a continuous and increasing function with $g(0)=0$. Using the Hölder inequality, we have

$$
\begin{aligned}
|(\mathscr{L} u)(t)-(\mathscr{L} u)(\tau)| \leq & \|f\|_{F}\left\{\frac{2}{\rho^{\alpha-1} \Gamma(\alpha)}\left[\left\|\mathscr{G}_{1}\right\|_{F^{*}}+\left\|\mathscr{G}_{2}\right\|_{F^{*}}\right]\right. \\
& \left.+\frac{\left|t^{3 \rho}-\tau^{3 \rho}\right|}{\rho^{3}|\psi|}\left[\sum_{i=1}^{m} \delta_{i} \frac{\xi_{i}^{\rho(\alpha+\beta)}}{\rho^{\alpha+\beta} \Gamma(\alpha+\beta+1)}-\frac{1}{\rho^{\alpha} \Gamma(\alpha+1)(\rho \alpha+1)}\right]\right\} .
\end{aligned}
$$

Then, $0<\left|t^{\rho}-\tau^{\rho}\right|<\epsilon$ and by the continuity of $g$, we see that $\mathscr{L} u$ is continuous, which completes the proof.
Our first existence result relies on Schaefer's fixed point theorem. 
6

Discrete Dynamics in Nature and Society

Theorem 5. Assume that there exists $\mathscr{M} \in C([0,1], \mathbb{R})$ such that

$$
\begin{aligned}
& \left(H_{1}\right)\left|f\left(t, u(t),{ }^{C} D_{0^{+}}^{\alpha-1, \rho} u(t),{ }^{C} D_{0^{+}}^{\alpha-2, \rho} u(t),{ }^{C} D_{0^{+}}^{\alpha-3, \rho} u(t)\right)\right| \\
& \quad \leq \mathscr{M}(t), \quad \text { for } t \in[0,1],
\end{aligned}
$$

Proof. The proof proceeds in few steps as follows:

Step 1: we will prove the operator $\mathscr{L}$ maps bounded sets into bounded sets in $C([0,1], \mathbb{R})$. Let $E \subset \mathrm{X}$ be a bounded set. Then, for all $u \in E$, by using the assumption $\left(H_{1}\right)$, for $t \in[0,1]$, we are able to obtain

with\| $\mathscr{M} \|=\max _{t \in[0,1]}|\mathscr{M}(t)|$. Then, the GFBVP (1) has at least one solution on $[0,1]$.

$$
\begin{aligned}
& |\mathscr{L} u(t)| \leq \frac{\rho^{1-\alpha}}{\Gamma(\alpha)} \int_{0}^{t}\left(t^{\rho}-s^{\rho}\right)^{\alpha-1} s^{\rho-1}\left|f\left(s, u(s),{ }^{C} D_{0^{+}}^{\alpha-1, \rho} u(s),{ }^{C} D_{0^{+}}^{\alpha-2, \rho} u(s),{ }^{C} D_{0^{+}}^{\alpha-3, \rho} u(s)\right)\right| \mathrm{d} s \\
& +\frac{\left|t^{3 \rho}\right|}{\rho^{3}|\psi|}\left[\sum_{i=1}^{m} \delta_{i} \frac{\rho^{1-(\alpha+\beta)}}{\Gamma(\alpha+\beta)} \int_{0}^{\xi_{i}}\left(\xi_{i}^{\rho}-s^{\rho}\right)^{\alpha+\beta-1} s^{\rho-1}\right. \\
& \cdot\left|f\left(s, u(s),{ }^{C} D_{0^{+}}^{\alpha-1, \rho} u(s),{ }^{C} D_{0^{+}}^{\alpha-2, \rho} u(s),{ }^{C} D_{0^{+}}^{\alpha-3, \rho} u(s)\right)\right| \mathrm{d} s \\
& \left.-\int_{0}^{1} I^{\alpha, \rho}\left|f\left(s, u(s),{ }^{C} D_{0^{+}}^{\alpha-1, \rho} u(s),{ }^{C} D_{0^{+}}^{\alpha-2, \rho} u(s),{ }^{C} D_{0^{+}}^{\alpha-3, \rho} u(s)\right)\right| \mathrm{d} s\right] \\
& \leq \frac{\rho^{1-\alpha}}{\Gamma(\alpha)} \int_{0}^{t}\left(t^{\rho}-s^{\rho}\right)^{\alpha-1} s^{\rho-1}|\mathscr{M}(s)| \mathrm{d} s \\
& +\frac{1}{\rho^{3}|\psi|}\left[\sum_{i=1}^{m} \delta_{i} \frac{\rho^{1-(\alpha+\beta)}}{\Gamma(\alpha+\beta)} \int_{0}^{\xi_{i}}\left(\xi_{i}^{\rho}-s^{\rho}\right)^{\alpha+\beta-1} s^{\rho-1}|\mathscr{M}(s)| \mathrm{d} s\right. \\
& \left.-\frac{\rho^{1-\alpha}}{\Gamma(\alpha)} \int_{0}^{1}\left(\int_{0}^{s}\left(s^{\rho}-\tau^{\rho}\right)^{\alpha-1} \tau^{\rho-1}|\mathscr{M}(\tau)| \mathrm{d} \tau\right) \mathrm{d} s\right] \\
& \leq \frac{\rho^{\alpha-1}\|\mathscr{M}\|}{\Gamma(\alpha)} \int_{0}^{t}\left(t^{\rho}-s^{\rho}\right)^{\alpha-1} s^{\rho-1} \mathrm{~d} s \\
& +\frac{1}{\rho^{3}|\psi|}\left[\sum_{i=1}^{m} \delta_{i}(\alpha+\beta) \int_{0}^{\xi_{i}}\left(\xi_{i}^{\rho}-s^{\rho}\right)^{\alpha+\beta-1} s^{\rho-1} \mathrm{~d} s\right. \\
& \left.-\frac{\|\mathscr{M}\|}{\rho^{\alpha} \Gamma(\alpha+1)(\rho \alpha+1)}\right] .
\end{aligned}
$$

Thus, we obtain

$$
\begin{aligned}
\|\mathscr{L} u\| & \leq\|\mathscr{M}\|\left\{\frac{1}{\rho^{\alpha} \Gamma(\alpha+1)}+\frac{1}{\rho^{3}|\psi|}\left[\sum_{i=1}^{m} \delta_{i} \frac{\xi_{i}^{\rho(\alpha+\beta)}}{\rho^{\alpha+\beta} \Gamma(\alpha+\beta+1)}-\frac{1}{\rho^{\alpha} \Gamma(\alpha+1)(\rho \alpha+1)}\right]\right\} \\
& =\mu_{1} .
\end{aligned}
$$


Discrete Dynamics in Nature and Society

7

Now, with some efforts of computation, we have

$$
\begin{aligned}
& \left({ }^{C} D_{0^{+}}^{\alpha-1, \rho} \mathscr{L} u\right)(t)=\left(I^{1, \rho} f\right)(t) \\
& +{ }^{C} D_{0^{+}}^{\alpha-1, \rho}\left\{\frac { t ^ { 3 \rho } } { \rho ^ { 3 } \psi } \left[\sum_{i=1}^{m} \delta_{i} \frac{\rho^{1-(\alpha+\beta)}}{\Gamma(\alpha+\beta)} \int_{0}^{\xi_{i}}\left(\xi_{i}^{\rho}-s^{\rho}\right)^{\alpha+\beta-1} s^{\rho-1}\right.\right. \\
& \cdot f\left(s, u(s),{ }^{C} D_{0^{+}}^{\alpha-1, \rho} u(s),{ }^{C} D_{0^{+}}^{\alpha-2, \rho} u(s),{ }^{C} D_{0^{+}}^{\alpha-3, \rho} u(s)\right) \mathrm{d} s \\
& \left.\left.-\int_{0}^{1} I^{\alpha, \rho} f\left(s, u(s),{ }^{C} D_{0^{+}}^{\alpha-1, \rho} u(s),{ }^{C} D_{0^{+}}^{\alpha-2, \rho} u(s),{ }^{C} D_{0^{+}}^{\alpha-3, \rho} u(s)\right) \mathrm{d} s\right]\right\} \\
& =\int_{0}^{t} s^{\rho-1} f\left(s, u(s),{ }^{C} D_{0^{+}}^{\alpha-1, \rho} u(s),{ }^{C} D_{0^{+}}^{\alpha-2, \rho} u(s),{ }^{C} D_{0^{+}}^{\alpha-3, \rho} u(s)\right) \mathrm{d} s \\
& +\frac{6 t^{\rho(4-\alpha)}}{\rho^{4-\alpha} \psi \Gamma(5-\alpha)}\left[\sum_{i=1}^{m} \delta_{i} \frac{\rho^{1-(\alpha+\beta)}}{\Gamma(\alpha+\beta)} \int_{0}^{\xi_{i}}\left(\xi_{i}^{\rho}-s^{\rho}\right)^{\alpha+\beta-1} s^{\rho-1}\right. \\
& \cdot f\left(s, u(s),{ }^{C} D_{0^{+}}^{\alpha-1, \rho} u(s),{ }^{C} D_{0^{+}}^{\alpha-2, \rho} u(s),{ }^{C} D_{0^{+}}^{\alpha-3, \rho} u(s)\right) \mathrm{d} s \\
& \left.-\int_{0}^{1} I^{\alpha, \rho} f\left(s, u(s),{ }^{C} D_{0^{+}}^{\alpha-1, \rho} u(s),{ }^{C} D_{0^{+}}^{\alpha-2, \rho} u(s),{ }^{C} D_{0^{+}}^{\alpha-3, \rho} u(s)\right) \mathrm{d} s\right] \\
& \leq \int_{0}^{t} s^{\rho-1} f\left(s, u(s),{ }^{C} D_{0^{+}}^{\alpha-1, \rho} u(s),{ }^{C} D_{0^{+}}^{\alpha-2, \rho} u(s),{ }^{C} D_{0^{+}}^{\alpha-3, \rho} u(s)\right) \mid \mathrm{d} s \\
& +\frac{6 t^{\rho(4-\alpha)}}{\rho^{4-\alpha} \psi \Gamma(5-\alpha)}\left[\sum_{i=1}^{m} \delta_{i} \frac{\rho^{1-(\alpha+\beta)}}{\Gamma(\alpha+\beta)} \int_{0}^{\xi_{i}}\left(\xi_{i}^{\rho}-s^{\rho}\right)^{\alpha+\beta-1} s^{\rho-1}\right. \\
& \cdot\left|f\left(s, u(s),{ }^{C} D_{0^{+}}^{\alpha-1, \rho} u(s),{ }^{C} D_{0^{+}}^{\alpha-2, \rho} u(s),{ }^{C} D_{0^{+}}^{\alpha-3, \rho} u(s)\right)\right| \mathrm{d} s \\
& -\frac{\rho^{1-\alpha}}{\Gamma(\alpha)} \int_{0}^{1}\left(\int_{0}^{s}\left(s^{\rho}-\tau^{\rho}\right)^{\alpha-1} \tau^{\rho-1}\right. \\
& \left.\left.\cdot\left|f\left(\tau, u(\tau),{ }^{C} D_{0^{+}}^{\alpha-1, \rho} u(\tau),{ }^{C} D_{0^{+}}^{\alpha-2, \rho} u(\tau),{ }^{C} D_{0^{+}}^{\alpha-3, \rho} u(\tau)\right) \tau^{\rho-1}\right| \mathrm{d} \tau\right) \mathrm{d} s\right] .
\end{aligned}
$$


Therefore, we have

$$
\begin{aligned}
& \left\|{ }^{C} D_{0^{+}}^{\alpha-1, \rho} \mathscr{L} u\right\| \leq\|\mathscr{M}\| \int_{0}^{t} s^{\rho-1} \mathrm{~d} s \\
& +\frac{6 t^{\rho(4-\alpha)}}{|\psi| \rho^{4-\alpha} \Gamma(5-\alpha)}\left[\sum_{i=1}^{m} \delta_{i} \frac{\|\mathscr{M}\| \rho^{1-(\alpha+\beta)}}{\Gamma(\alpha+\beta)}\right. \\
& \left.\cdot \int_{0}^{\xi_{i}}\left(\xi_{i}^{\rho}-s^{\rho}\right)^{\alpha+\beta-1} s^{\rho-1} d s-\frac{\|\mathscr{M}\|}{\rho^{\alpha} \Gamma(\alpha+1)(\rho \alpha+1)}\right] \\
& \leq \frac{\|\mathscr{M}\| t^{\rho}}{\rho}+\frac{6 t^{\rho(4-\alpha)}}{|\psi| \rho^{4-\alpha} \Gamma(5-\alpha)}\left[\sum_{i=1}^{m} \delta_{i} \frac{\|\mathscr{M}\| \xi_{i}^{\rho(\alpha+\beta)}}{\rho^{\alpha+\beta} \Gamma(\alpha+\beta+1)}\right. \\
& \left.\quad-\frac{\|\mathscr{M}\|}{\rho^{\alpha} \Gamma(\alpha+1)(\rho \alpha+1)}\right] \\
& \leq\|\mathscr{M}\|\left\{\frac{1}{\rho}+\frac{6}{|\psi| \rho^{4-\alpha} \Gamma(5-\alpha)}\left[\sum_{i=1}^{m} \delta_{i} \frac{\xi_{i}^{\rho(\alpha+\beta)}}{\rho^{\alpha+\beta} \Gamma(\alpha+\beta+1)}\right.\right. \\
& \left.\left.-\frac{1}{\rho^{\alpha} \Gamma(\alpha+1)(\rho \alpha+1)}\right]\right\}=\mu_{2} .
\end{aligned}
$$

In a similar way, we arrive at

$$
\begin{aligned}
\left\|{ }^{C} D_{0^{+}}^{\alpha-2} \mathscr{L} u\right\| \leq & \|\mathscr{M}\|\left\{\frac{1}{\rho^{2} \Gamma(3)}+\frac{6}{|\psi| \rho^{5-\alpha} \Gamma(6-\alpha)}\right. \\
& \cdot\left[\sum_{i=1}^{m} \delta_{i} \frac{\xi_{i}^{\rho(\alpha+\beta)}}{\rho^{\alpha+\beta} \Gamma(\alpha+\beta+1)}\right. \\
& \left.\left.-\frac{1}{\rho^{\alpha} \Gamma(\alpha+1)(\rho \alpha+1)}\right]\right\} \\
= & \mu_{3} .
\end{aligned}
$$

$$
\begin{aligned}
& \left.\left.-\frac{1}{\rho^{\alpha} \Gamma(\alpha+1)(\rho \alpha+1)}\right]\right\} \\
= & \mu_{4} .
\end{aligned}
$$

Step 2: from (32)-(36), we can immediately get the operator $\mathscr{L}: X \longrightarrow X$ to map bounded sets into bounded sets. Let $0<t_{1}<t_{2}<1$, and for all $u \in E$, we get

$$
\begin{aligned}
& \left|(\mathscr{L} u)\left(t_{2}\right)-(\mathscr{L} u)\left(t_{1}\right)\right| \\
& =\mid \frac{\rho^{1-\alpha}}{\Gamma(\alpha)} \int_{0}^{t_{2}}\left(t_{2}^{\rho}-s^{\rho}\right)^{\alpha-1} s^{\rho-1} \\
& f\left(s, u(s),{ }^{C} D_{0^{+}}^{\alpha-1, \rho} u(s),{ }^{C} D_{0^{+}}^{\alpha-2, \rho} u(s),{ }^{C} D_{0^{+}}^{\alpha-3, \rho} u(s)\right) \mathrm{d} s \\
& +\frac{\rho^{1-\alpha}}{\Gamma(\alpha)} \int_{0}^{t_{1}}\left(t_{1}^{\rho}-s^{\rho}\right)^{\alpha-1} s^{\rho-1} \\
& f\left(s, u(s),{ }^{C} D_{0^{+}}^{\alpha-1, \rho} u(s),{ }^{C} D_{0^{+}}^{\alpha-2, \rho} u(s),{ }^{C} D_{0^{+}}^{\alpha-3, \rho} u(s)\right) \mathrm{d} s \\
& +\frac{t_{2}^{3 \rho}-t_{1}^{3 \rho}}{\psi \rho^{3}}\left[\sum_{i=1}^{m} \delta_{i} \frac{\rho^{1-(\alpha+\beta)}}{\Gamma(\alpha+\beta)} \int_{0}^{\xi_{i}}\left(\xi_{i}^{\rho}-s^{\rho}\right)^{\alpha+\beta-1} s^{\rho-1}\right. \\
& f\left(s, u(s),{ }^{C} D_{0^{+}}^{\alpha-1, \rho} u(s),{ }^{C} D_{0^{+}}^{\alpha-2, \rho} u(s),{ }^{C} D_{0^{+}}^{\alpha-3, \rho} u(s)\right) \mathrm{d} s \\
& \left.-\int_{0}^{1} I^{\alpha, \rho} f\left(s, u(s),{ }^{C} D_{0^{+}}^{\alpha-1, \rho} u(s),{ }^{C} D_{0^{+}}^{\alpha-2, \rho} u(s),{ }^{C} D_{0^{+}}^{\alpha-3, \rho} u(s)\right) \mathrm{d} s\right] \mid \\
& \leq \frac{\rho^{1-\alpha}}{\Gamma(\alpha)} \int_{0}^{t_{1}}\left[\left(t_{2}^{\rho}-s^{\rho}\right)^{\alpha-1}-\left(t_{1}^{\rho}-s^{\rho}\right)^{\alpha-1}\right] s^{\rho-1} \\
& \cdot\left|f\left(s, u(s),{ }^{C} D_{0^{+}}^{\alpha-1, \rho} u(s),{ }^{C} D_{0^{+}}^{\alpha-2, \rho} u(s),{ }^{C} D_{0^{+}}^{\alpha-3, \rho} u(s)\right)\right| \mathrm{d} s \\
& +\int_{t_{1}}^{t_{2}}\left(t_{2}^{\rho}-s^{\rho}\right)^{\alpha-1} \\
& \left.\left|f\left(s, u(s),{ }^{C} D_{0^{+}}^{\alpha-1, \rho} u(s),{ }^{C} D_{0^{+}}^{\alpha-2, \rho} u(s),{ }^{C} D_{0^{+}}^{\alpha-3, \rho} u(s)\right)\right| \mathrm{d} s\right] \\
& +\frac{\left(t_{2}^{3 \rho}-t_{1}^{3 \rho}\right)}{|\psi| \rho^{3}}\left[\sum_{i=1}^{m} \delta_{i} \frac{\rho^{1-(\alpha+\beta)}}{\Gamma(\alpha+\beta)} \int_{0}^{\xi_{i}}\left(\xi_{i}^{\rho}-s^{\rho}\right)^{\alpha+\beta-1} s^{\rho-1}\right. \\
& \cdot\left|f\left(s, u(s),{ }^{C} D_{0^{+}}^{\alpha-1, \rho} u(s),{ }^{C} D_{0^{+}}^{\alpha-2, \rho} u(s),{ }^{C} D_{0^{+}}^{\alpha-3, \rho} u(s)\right)\right| \mathrm{d} s \\
& \left.-\int_{0}^{1} I^{\alpha, \rho}\left|f\left(s, u(s),{ }^{C} D_{0^{+}}^{\alpha-1, \rho} u(s),{ }^{C} D_{0^{+}}^{\alpha-2, \rho} u(s),{ }^{C} D_{0^{+}}^{\alpha-3, \rho} u(s)\right)\right| \mathrm{d} s\right] .
\end{aligned}
$$

Thus, we have

$$
\begin{aligned}
& \left|(\mathscr{L} u)\left(t_{2}\right)-(\mathscr{L} u)\left(t_{1}\right)\right| \\
& \leq\|\mathscr{M}\|\left\{\frac{1}{\rho^{\alpha} \Gamma(\alpha+1)}\left[2\left(t_{2}^{\rho}-t_{1}^{\rho}\right)^{\alpha}-\left(t_{2}^{\alpha \rho}-t_{1}^{\alpha \rho}\right)\right]\right. \\
& \quad+\frac{\left(t_{2}^{3 \rho}-t_{1}^{3 \rho}\right)}{|\psi| \rho^{3}}\left[\sum_{i=1}^{m} \delta_{i} \frac{\xi_{i}^{\rho(\alpha+\beta)}}{\rho^{\alpha+\beta} \Gamma(\alpha+\beta+1)}\right. \\
& \left.\left.-\frac{1}{\rho^{\alpha} \Gamma(\alpha+1)(\rho \alpha+1)}\right]\right\} .
\end{aligned}
$$

Similarly, it can be easily shown that 


$$
\begin{aligned}
& \left|\left({ }^{C} D_{0^{+}}^{\alpha-1, \rho} \mathscr{L} u\right)\left(t_{2}\right)-\left({ }^{C} D_{0^{+}}^{\alpha-1, \rho} \mathscr{L} u\right)\left(t_{1}\right)\right| \leq\|\mathscr{M}\|\left\{\frac{\left(t_{2}^{\rho}-t_{1}^{\rho}\right)}{\rho}+\frac{6\left(t_{2}^{\rho(4-\alpha)}-t_{1}^{\rho(4-\alpha)}\right)}{|\psi| \rho^{4-\alpha} \Gamma(5-\alpha)}\right. \\
& \left.\left[\sum_{i=1}^{m} \delta_{i} \frac{\xi_{i}^{\rho(\alpha+\beta)}}{\rho^{\alpha+\beta} \Gamma(\alpha+\beta+1)}-\frac{1}{\rho^{\alpha} \Gamma(\alpha+1)(\rho \alpha+1)}\right]\right\} \text {. } \\
& \left|\left({ }^{C} D_{0^{+}}^{\alpha-2, \rho} \mathscr{L} u\right)\left(t_{2}\right)-\left({ }^{C} D_{0^{+}}^{\alpha-2, \rho} \mathscr{L} u\right)\left(t_{1}\right)\right| \leq\|\mathscr{M}\|\left\{\frac{\left(t_{2}^{2 \rho}-t_{1}^{2 \rho}\right)}{\rho^{2} \Gamma(3)}+\frac{6\left(t_{2}^{\rho(5-\alpha)}-t_{1}^{\rho(5-\alpha)}\right)}{|\psi| \rho^{5-\alpha} \Gamma(6-\alpha)}\right. \\
& \left.\cdot\left[\sum_{i=1}^{m} \delta_{i} \frac{\xi_{i}^{\rho(\alpha+\beta)}}{\rho^{\alpha+\beta} \Gamma(\alpha+\beta+1)}-\frac{1}{\rho^{\alpha} \Gamma(\alpha+1)(\rho \alpha+1)}\right]\right\} \text {. } \\
& \left|\left({ }^{C} D_{0^{+}}^{\alpha-3, \rho} \mathscr{L} u\right)\left(t_{2}\right)-\left({ }^{C} D_{0^{+}}^{\alpha-3, \rho} \mathscr{L} u\right)\left(t_{1}\right)\right| \leq\|\mathscr{M}\|\left\{\frac{\left(t_{2}^{3 \rho}-t_{1}^{3 \rho}\right)}{\rho^{3} \Gamma(4)}+\frac{6\left(t_{2}^{\rho(6-\alpha)}-t_{1}^{\rho(6-\alpha)}\right)}{|\psi| \rho^{6-\alpha} \Gamma(7-\alpha)}\right. \\
& \left.\cdot\left[\sum_{i=1}^{m} \delta_{i} \frac{\xi_{i}^{\rho(\alpha+\beta)}}{\rho^{\alpha+\beta} \Gamma(\alpha+\beta+1)}-\frac{1}{\rho^{\alpha} \Gamma(\alpha+1)(\rho \alpha+1)}\right]\right\} .
\end{aligned}
$$

The right side of (38)-(41) goes to zero as $\left(t_{2}-t_{1}\right) \longrightarrow 0$. In view of steps I and II, it follows that by Arzela-Ascoli theorem, the sets $\{\mathscr{L}(u): u \in E\},\left\{{ }^{C} D_{0^{+}}^{\alpha-1, \rho} \mathscr{L} u: u \in\right.$ $E\},\left\{{ }^{C} D_{0^{+}}^{\alpha-2, \rho} \mathscr{L} u \mathscr{L}(u): u \in E\right\}$, and $\left\{{ }^{C} D_{0^{+}}^{\alpha-3, \rho} \mathscr{L}(u): u \in E\right\}$ are relatively compact in $C([0,1])$. Therefore, $\mathscr{L}(E)$ is a relatively compact set in $X$. We consider the set $T=\{u \in \mathrm{X} ; u=\lambda \mathscr{L} u, 0<\lambda<1\}$. Then, $T$ is bounded. Indeed, let $u \in T$. So, $u=\lambda \mathscr{L} u, 0<\lambda<1$, for any $t \in[0,1]$, and it follows that

$$
\begin{aligned}
\|u\| \leq & \frac{\|\mathscr{M}\|}{\rho^{\alpha} \Gamma(\alpha+1)}+\frac{1}{\rho^{3}|\psi|}\left[\sum_{i=1}^{m} \delta_{i} \frac{\|\mathscr{M}\| \xi_{i}^{\rho(\alpha+\beta)}}{\rho^{\alpha+\beta} \Gamma(\alpha+\beta+1)}\right. \\
& \left.-\frac{\|\mathscr{M}\|}{\rho^{\alpha} \Gamma(\alpha+1)(\rho \alpha+1)}\right] .
\end{aligned}
$$

Thus, all the hypotheses of Theorem 3 are satisfied. Therefore, we can conclude that the operator $\mathscr{L}$ has at least one fixed point. Hence, the GFBVP (1) has at least one solution on $[0,1]$.

For computation convenience, we set

$$
\begin{aligned}
V & =\sum_{i=1}^{m} \delta_{i} \frac{\xi_{i}^{\rho(\alpha+\beta)}}{\rho^{\alpha+\beta} \Gamma(\alpha+\beta+1)}-\frac{1}{\rho^{\alpha} \Gamma(\alpha+1)(\rho \alpha+1)}, \\
K & =\frac{6}{\rho^{4-\alpha} \psi \Gamma(5-\alpha)}, \\
\sigma & =\frac{1}{\rho} .
\end{aligned}
$$

Now, we make use of Theorem 4 to prove the existence of solutions of GFBVP (1).

Theorem 6. Let $f:[0,1] \times \mathbb{R}^{4} \longrightarrow \mathbb{R}$ be a continuous function such that the following assumptions hold:

$$
\begin{aligned}
&\left(H_{2}\right)|f(t, u, p, q, w)-f(t, v, \tilde{p}, \tilde{q}, \widetilde{w})| \leq L(|u-v|+|p-\tilde{p}|+|q-\tilde{q}|+|w-\widetilde{w}|), \quad \text { for all } t \in[0,1], u, v, p, q, w, \tilde{p}, \tilde{q}, \widetilde{w} \in R \text { and } L>0, \\
&\left(H_{3}\right)\left|f\left(t, u(t),{ }^{C} D_{0^{+}}^{\alpha-1, \rho} u(t),{ }^{C} D_{0^{+}}^{\alpha-2, \rho} u(t),{ }^{C} D_{0^{+}}^{\alpha-3, \rho} u(t)\right)\right| \leq \phi(t),
\end{aligned}
$$

for $t \in[0,1]$ and $\phi \in C([0,1], \mathbb{R})$ with $\|\phi\|=\max _{t \in[0,1]}|\phi(t)|$.

Then, GFBVP (1) has at least one solution on $[0,1]$, provided that

$$
L K V<1 .
$$

Proof. We define $B_{r}=\{u \in X:\|u\| \leq r\}$, where $r \geqslant\|\phi\|(\sigma+$ $K V)$. We split the operator $\mathscr{L}$ defined by (21) on $B_{r}$ as $\mathscr{L}=\mathscr{L}_{1}+\mathscr{L}_{2}$, where $\mathscr{L}_{1}$ and $\mathscr{L}_{2}$ are given by 


$$
\begin{aligned}
\left(\mathscr{L}_{1} u\right)(t)= & \frac{\rho^{1-\alpha}}{\Gamma(\alpha)} \int_{0}^{t}\left(t^{\rho}-s^{\rho}\right)^{\alpha-1} s^{\rho-1} f\left(s, u(s),{ }^{C} D_{0^{+}}^{\alpha-1, \rho} u(s),{ }^{C} D_{0^{+}}^{\alpha-2, \rho} u(s),{ }^{C} D_{0^{+}}^{\alpha-3, \rho} u(s)\right) \mathrm{d} s \\
\left(\mathscr{L}_{2} u\right)(t)= & \frac{t^{3 \rho}}{\rho^{3} \psi}\left[\sum_{i=1}^{m} \delta_{i} \frac{\rho^{1-(\alpha+\beta)}}{\Gamma(\alpha+\beta)} \int_{0}^{\xi_{i}}\left(\xi_{i}^{\rho}-s^{\rho}\right){ }^{\alpha+\beta-1} s^{\rho-1}\right. \\
& \cdot f\left(s, u(s),{ }^{C} D_{0^{+}}^{\alpha-1, \rho} u(s),{ }^{C} D_{0^{+}}^{\alpha-2, \rho} u(s),{ }^{C} D_{0^{+}}^{\alpha-3, \rho} u(s)\right) \mathrm{d} s \\
& \left.-\int_{0}^{1} I^{\alpha, \rho} f\left(s, u(s),{ }^{C} D_{0^{+}}^{\alpha-1, \rho} u(s),{ }^{C} D_{0^{+}}^{\alpha-2, \rho} u(s),{ }^{C} D_{0^{+}}^{\alpha-3, \rho} u(s)\right) \mathrm{d} s\right]
\end{aligned}
$$

For $u, v \in B_{r}$, we find that

$$
\begin{aligned}
& \left\|\mathscr{L}_{1}(u)+\mathscr{L}_{2}(u)\right\| \leq \max _{t \in[0,1]}\left\{\frac{\rho^{1-\alpha}}{\Gamma(\alpha)} \int_{0}^{t}\left(t^{\rho}-s^{\rho}\right)^{\alpha-1} s^{\rho-1}\right. \\
& \cdot\left|f\left(s, u(s),{ }^{C} D_{0^{+}}^{\alpha-1, \rho}, u(s),{ }^{C} D_{0^{+}}^{\alpha-2, \rho} u(s),{ }^{C} D_{0^{+}}^{\alpha-3, \rho} u(s)\right)\right| \mathrm{d} s \\
& +\frac{t^{3 \rho}}{\rho^{3} \psi}\left[\sum_{i=1}^{m} \delta_{i} \frac{\rho^{1-(\alpha+\beta)}}{\Gamma(\alpha+\beta)} \int_{0}^{\xi_{i}}\left(\xi_{i}^{\rho}-s^{\rho}\right)^{\alpha+\beta-1} s^{\rho-1}\right. \\
& \cdot\left|f\left(s, u(s),{ }^{C} D_{0^{+}}^{\alpha-1, \rho}, u(s),{ }^{C} D_{0^{+}}^{\alpha-2, \rho} u(s),{ }^{C} D_{0^{+}}^{\alpha-3, \rho} u(s)\right)\right| \mathrm{d} s \\
& \left.\left.-\int_{0}^{1} I^{\alpha, \rho}\left|f\left(s, u(s),{ }^{C} D_{0^{+}}^{\alpha-1, \rho}, u(s),{ }^{C} D_{0^{+}}^{\alpha-2, \rho} u(s),{ }^{C} D_{0^{+}}^{\alpha-3, \rho} u(s)\right)\right| \mathrm{d} s\right]\right\} \\
& \leq\|\phi\|\left[\frac{1}{\rho^{\alpha} \Gamma(\alpha+1)}+\frac{1}{\rho^{3}|\psi|}\left(\sum_{i=1}^{m} \delta_{i} \frac{\xi_{i}^{\rho(\alpha+\beta)}}{\rho^{\alpha+\beta \Gamma(\alpha+\beta+1)}}\right.\right. \\
& \left.\left.-\frac{1}{\rho^{\alpha} \Gamma(\alpha+1)(\rho \alpha+1)}\right)\right] \\
& \leq\|\phi\|(\sigma+K V) \leq r .
\end{aligned}
$$

\section{Furthermore,}


Discrete Dynamics in Nature and Society

11

$$
\begin{aligned}
\left\|\left({ }^{C} D_{0^{+}}^{\alpha-1, \rho} \mathscr{L}_{1}\right)(u)+\left({ }^{C} D_{0^{+}}^{\alpha-1, \rho} \mathscr{L}_{2}\right)(u)\right\| \leq & \|\phi\| \\
& \left.\cdot\left(\sum_{i=1}^{m} \delta_{i} \frac{1}{\rho} \frac{\xi_{i}^{\rho+\beta} \Gamma(\alpha+\beta)}{|\psi| \rho^{4-\alpha} \Gamma(5-\alpha)}-\frac{1}{\rho^{\alpha} \Gamma(\alpha+1)(\rho \alpha+1)}\right)\right] \\
\leq & \|\phi\|(\sigma+K V) \leq r, \\
\left\|\left({ }^{C} D_{0^{+}}^{\alpha-2, \rho} \mathscr{L}_{1}\right)(u)+\left({ }^{C} D_{0^{+}}^{\alpha-2, \rho} \mathscr{L}_{2}\right)(u)\right\| \leq & \|\phi\|\left[\frac{1}{\rho^{2} \Gamma(3)}+\frac{6}{|\psi| \rho^{5-\alpha} \Gamma(6-\alpha)}\right. \\
& \left.\cdot\left(\sum_{i=1}^{m} \delta_{i} \frac{\xi_{i}^{\rho(\alpha+\beta)}}{\rho^{\alpha+\beta} \Gamma(\alpha+\beta+1)}-\frac{1}{\rho^{\alpha} \Gamma(\alpha+1)(\rho \alpha+1)}\right)\right] \\
\leq & \|\phi\|(\sigma+K V) \leq r, \\
\left\|\left({ }^{C} D_{0^{+}}^{\alpha-3, \rho} \mathscr{L}_{1}\right)(u)+\left({ }^{C} D_{0^{+}}^{\alpha-3, \rho} T_{3}\right)(u)\right\| \leq & \|\phi\|\left[\frac{1}{\rho^{3} \Gamma(4)}+\frac{6}{\|\psi\| \rho^{6-\alpha} \Gamma(7-\alpha)}\right. \\
& \left.\cdot\left(\sum_{i=1}^{m} \delta_{i} \frac{\xi_{i}^{\rho(\alpha+\beta)}}{\rho^{\alpha+\beta} \Gamma(\alpha+\beta+1)}-\frac{1}{\rho^{\alpha} \Gamma(\alpha+1)(\rho \alpha+1)}\right)\right] \\
\leq & \|\phi\|(\sigma+K V) \leq r .
\end{aligned}
$$

Consequently, we obtain

$$
\left\|\mathscr{L}_{1}(u)+\mathscr{L}_{2}(u)\right\|_{X} \leq\|\phi\|(\sigma+K V) \leq r,
$$

which shows that $\mathscr{L}_{1}(u)+\mathscr{L}_{2}(u) \in B_{r}$. In what follows, we prove that $\mathscr{L}_{2}$ is a contraction. Let $u, v \in B_{r}$ and for all $t \in[0,1]$, we get

$$
\begin{aligned}
& \left\|\mathscr{L}_{2}(u)-\mathscr{L}_{2}(v)\right\|=\sup _{t \in[0,1]}\left|\mathscr{L}_{2}(u)-\mathscr{L}_{2}(v)\right| \\
& =\sup _{t \in[0,1]} \mid \frac{t^{3} \rho}{\rho^{3} \psi} \sum_{i=1}^{m} \delta_{i} \frac{\xi_{i}^{\rho(\alpha+\beta)}}{\rho(\alpha+\beta)} \int_{0}^{\xi_{i}}\left(\xi_{i}^{p}-s^{p}\right)^{\alpha+\beta+1} s^{p-1} \\
& \cdot f\left(s, u(s),{ }^{C} D_{0^{+}}^{\alpha-1, \rho} u(s),{ }^{C} D_{0^{+}}^{\alpha-2, \rho},{ }^{C} D_{0^{+}}^{\alpha-3, \rho} u(s)\right) \mathrm{d} s \\
& -\int_{0}^{1} I^{\alpha, \rho} f\left(s, u(s),{ }^{C} D_{0^{+}}^{\alpha-1, \rho} u(s),{ }^{C} D_{0^{+}}^{\alpha-2, \rho} u(s),{ }^{C} D_{0^{+}}^{\alpha-3, \rho} u(s)\right) \mathrm{d} s \\
& -\frac{t^{3} \rho}{\rho^{3} \psi} \sum_{i=1}^{m} \delta_{i} \frac{\xi_{i}^{\rho(\alpha+\beta)}}{\rho(\alpha+\beta)} \int_{0}^{\xi_{i}}\left(\xi_{i}^{p}-s^{p}\right)^{\alpha+\beta+1} s^{p-1} \\
& \cdot f\left(s, u(s),{ }^{C} D_{0^{+}}^{\alpha-1, \rho} u(s),{ }^{C} D_{0^{+}}^{\alpha-2, \rho} u(s),{ }^{C} D_{0^{+}}^{\alpha-3, \rho} u(s)\right) \mathrm{d} s \\
& \left.+\int_{0}^{1} I^{\alpha, \rho} f\left(s, v(s),{ }^{C} D_{0^{+}}^{\alpha-1, \rho} v(s),{ }^{C} D_{0^{+}}^{\alpha-2, \rho} v(s),{ }^{C} D_{0^{+}}^{\alpha-3, \rho} v(s)\right) \mathrm{d} s\right] \mid \\
& =\sup _{t \in[0,1]} \mid \frac{t^{3} \rho}{\rho^{3} \psi}\left[\sum_{i=1}^{m} \delta_{i} \frac{\xi_{i}^{\rho(\alpha+\beta)}}{\rho(\alpha+\beta)} \int_{0}^{\xi_{i}}\left(\xi_{i}^{p}-s^{p}\right)^{\alpha+\beta+1} s^{p-1}\right.
\end{aligned}
$$




$$
\begin{aligned}
& \cdot f\left(s, u(s),{ }^{C} D_{0^{+}}^{\alpha-1, \rho} u(s),{ }^{C} D_{0^{+}}^{\alpha-2, \rho} u(s),{ }^{C} D_{0^{+}}^{\alpha-3, \rho} u(s)\right. \\
& \left.-f\left(s, u(s),{ }^{C} D_{0^{+}}^{\alpha-1, \rho} u(s),{ }^{C} D_{0^{+}}^{\alpha-2, \rho} u(s),{ }^{C} D_{0^{+}}^{\alpha-3, \rho} u(s)\right)\right) d s \\
& -\int_{0}^{1} I^{\alpha, \rho}\left(f\left(s, u(s),{ }^{C} D_{0^{+}}^{\alpha-1, \rho} u(s),{ }^{C} D_{0^{+}}^{\alpha-2, \rho} u(s),{ }^{C} D_{0^{+}}^{\alpha-3, \rho} u(s)\right)\right. \\
& \left.\left.-f\left(s, u(s),{ }^{C} D_{0^{+}}^{\alpha-1, \rho} u(s),{ }^{C} D_{0^{+}}^{\alpha-2, \rho} u(s),{ }^{C} D_{0^{+}}^{\alpha-3, \rho} u(s)\right)\right) d s\right] \mid \\
\leq & \sup _{t \in[0,1]}\left[L \left(|u(t)-v(t)|+\left|{ }^{C} D_{0^{+}}^{\alpha-1, \rho} u(t)-{ }^{C} D_{0^{+}}^{\alpha-1, \rho} v(t)\right|\right.\right. \\
& \left.\left.+\left|{ }^{C} D_{0^{+}}^{\alpha-2, \rho} u(t)-{ }^{C} D_{0^{+}}^{\alpha-2, \rho} v(t)\right|+\left|{ }^{C} D_{0^{+}}^{\alpha-3, \rho} u(t)-{ }^{C} D_{0^{+}}^{\alpha-3, \rho} v(t)\right|\right)\right] \\
\leq & L K V\|u-v\| . \\
& \cdot\left(\frac{t^{3} \rho}{\rho^{3} \psi} \sum_{i=1}^{m} \delta_{i} \frac{\xi_{i}^{\rho(\alpha+\beta)}}{\rho^{\alpha+\beta} \Gamma(\alpha+\beta+1)} \int_{0}^{\xi_{i}}\left(\xi_{i}^{p}-s^{p}\right)^{\alpha+\beta+1} s^{p^{-1}} d s-\frac{1}{\rho^{\alpha} \Gamma(\alpha+1)(\rho \alpha+1)}\right) \\
& {\left[\frac{1}{\rho^{3} \psi} \sum_{i=1}^{m} \delta_{i} \frac{\xi_{i}^{\rho(\alpha+\beta)}}{\rho^{\alpha+\beta} \Gamma(\alpha+\beta+1)}-\frac{1}{\rho^{\alpha} \Gamma(\alpha+1)(\rho \alpha+1)}\right]\|u-v\| } \\
\leq & 1
\end{aligned}
$$

With the same process, one has

$$
\begin{gathered}
\left\|{ }^{C} D_{0^{+}}^{\alpha-1, \rho} \mathscr{L}_{2}(u)-{ }^{C C} D_{0^{+}}^{\alpha-1, \rho} \mathscr{L}_{2}(v)\right\| \leq L K V\|u-v\|, \\
\left\|{ }^{C} D_{0^{+}}^{\alpha-2, \rho} \mathscr{L}_{2}(u)-{ }^{C} D_{0^{+}}^{\alpha-2, \rho} \mathscr{L}_{2}(v)\right\| \leq L K V\|u-v\|, \\
\left\|{ }^{C} D_{0^{+}}^{\alpha-3, \rho} \mathscr{L}_{2}(u)-{ }^{C} D_{0^{+}}^{\alpha-3, \rho} \mathscr{L}_{2}(v)\right\| \leq L K V\|u-v\| .
\end{gathered}
$$

Hence, we get

$$
\left\|\mathscr{L}_{2}(u)-\mathscr{L}_{2}(v)\right\|_{X} \leq L K V\|u-v\|_{X}
$$

It remains to show that $\mathscr{L}_{1}$ is continuous and compact. The continuity of the function $f$ implies that the operator $\mathscr{L}_{1}$ is continuous. To achieve the compactness of the operator $\mathscr{L}_{1}$, we first prove that $\mathscr{L}_{1}$ is uniformly bounded on $B_{r}$ as follows:

$$
\begin{aligned}
\left\|\mathscr{L}_{1}(u)\right\|= & \frac{\rho^{1-\alpha}}{\Gamma(\alpha)} \sup _{t \in[0,1]} \int_{0}^{t}\left(t^{\rho}-s^{\rho}\right)^{\alpha-1} s^{\rho-1} \\
& \cdot\left|f\left(s, u(s),{ }^{C} D_{0^{+}}^{\alpha-1, \rho} u(s),{ }^{C} D_{0^{+}}^{\alpha-2, \rho} u(s),{ }^{C} D_{0^{+}}^{\alpha-3, \rho} u(s)\right)\right| \mathrm{d} s \\
\leq & \frac{\|\phi\|}{\rho^{\alpha} \Gamma(\alpha+1)} \leq\|\phi\| \sigma, \\
\left\|\left({ }^{C} D_{0^{+}}^{\alpha-1, \rho} \mathscr{L}_{1}\right)(u)\right\|= & \sup _{t \in[0,1]}\left(\frac{\|\phi\| t^{\rho}}{\rho}\right) \leq \frac{\|\phi\|}{\rho} \leq\|\phi\| \sigma, \\
\left\|\left({ }^{C} D_{0^{+}}^{\alpha-2, \rho} \mathscr{L}_{1}\right)(u)\right\|= & \sup _{t \in[0,1]}\left(\frac{\|\phi\| t^{2 \rho}}{\rho^{2} \Gamma(3)}\right) \leq \frac{\|\phi\|}{\rho^{2} \Gamma(3)} \leq\|\phi\| \sigma, \\
\left\|\left({ }^{C} D_{0^{+}}^{\alpha-3, \rho} \mathscr{L}_{1}\right)(u)\right\|= & \sup _{t \in[0,1]}\left(\frac{\|\phi\| t^{3 \rho}}{\rho^{3} \Gamma(4)}\right) \leq \frac{\|\phi\|}{\rho^{3} \Gamma(4)} \leq\|\phi\| \sigma .
\end{aligned}
$$


So,

$$
\left\|\mathscr{L}_{1}(u)\right\|_{X} \leq\|\phi\| \sigma .
$$

Now, let $\sup _{t \in[0,1] \times B_{r}^{4}}|f(t, u, p, q, w)|=\eta$. Consequently, for $t_{1}, t_{2} \in[0,1], t_{1}<t_{2}$, we have

$$
\left|\left(\mathscr{L}_{1} u\right)\left(t_{2}\right)-\left(\mathscr{L}_{1} u\right)\left(t_{1}\right)\right| \leq \frac{\eta}{\rho}\left[2\left(t_{2}^{\rho}-t_{1}^{\rho}\right)^{\alpha}-\left(t_{2}^{\alpha \rho}-t_{1}^{\alpha \rho}\right)\right] .
$$

Furthermore,

$$
\begin{aligned}
& \left|\left({ }^{C} D_{0^{+}}^{\alpha-1, \rho} \mathscr{L}_{1} u\right)\left(t_{2}\right)-\left({ }^{C} D_{0^{+}}^{\alpha-1, \rho} \mathscr{L}_{1} u\right)\left(t_{1}\right)\right| \leq \frac{\eta}{\rho}\left|t_{2}^{\rho}-t_{1}^{\rho}\right|, \\
& \left|\left({ }^{C} D_{0^{+}}^{\alpha-2, \rho} \mathscr{L}_{1} u\right)\left(t_{2}\right)-\left({ }^{C} D_{0^{+}}^{\alpha-2, \rho} \mathscr{L}_{1} u\right)\left(t_{1}\right)\right| \leq \frac{\eta}{\rho}\left|t_{2}^{2 \rho}-t_{1}^{2 \rho}\right|, \\
& \left|\left({ }^{C} D_{0^{+}}^{\alpha-2, \rho} \mathscr{L}_{1} u\right)\left(t_{2}\right)-\left({ }^{C} D_{0^{+}}^{\alpha-2, \rho} \mathscr{L}_{1} u\right)\left(t_{1}\right)\right| \leq \frac{\eta}{\rho}\left|t_{2}^{3 \rho}-t_{1}^{3 \rho}\right|,
\end{aligned}
$$

Therefore, as $\left(t_{2}-t_{1}\right) \longrightarrow 0$, the right-hand sides of the above inequalities tend to zero independently of $u \in B_{r}$. Thus, $\mathscr{L}_{1}$ is equicontinuous and so it is relatively compact on $B_{r}$. According to the Arzela-Ascoli theorem, the operator $\mathscr{L}_{1}$ is compact. By using Theorem 4 , there exists at least one solution of GFBVP (1) on $[0,1]$.

In the next result, we prove the existence of solutions for GFBVP (1) by applying Banach's fixed point theorem.

Theorem 7. Assume that the condition $\left(\mathrm{H}_{2}\right)$ holds. Then, GFBVP (1) has a unique solution on $[0,1]$, provided that

$$
L A<1 \text {, }
$$

where $A=\sigma+K V$ and $V, K$, and $\sigma$ are given by (43)-(45).

Proof. Consider the operator $\mathscr{L}: X \longrightarrow X$ defined by

$$
\begin{aligned}
(\mathscr{L} u)(t)= & \frac{\rho^{1-\alpha}}{\Gamma(\alpha)} \int_{0}^{t}\left(t^{\rho}-s^{\rho}\right)^{\alpha-1} s^{\rho-1} f\left(s, u(s),{ }^{C} D_{0^{+}}^{\alpha-1, \rho} u(s),{ }^{C} D_{0^{+}}^{\alpha-2, \rho} u(s),{ }^{C} D_{0^{+}}^{\alpha-3, \rho} u(s)\right) \mathrm{d} s \\
& +\frac{t^{3 \rho}}{\rho^{3} \psi}\left[\sum_{i=1}^{m} \delta_{i} \frac{\rho^{1-(\alpha+\beta)} \Gamma(\alpha+\beta)}{\Gamma} \int_{0}^{\xi_{i}}\left(\xi_{i}^{\rho}-s^{\rho}\right){ }^{\alpha+\beta-1} s^{\rho-1}\right. \\
& \cdot f\left(s, u(s),{ }^{C} D_{0^{+}}^{\alpha-1, \rho} u(s),{ }^{C} D_{0^{+}}^{\alpha-2, \rho} u(s),{ }^{C} D_{0^{+}}^{\alpha-3, \rho} u(s)\right) \mathrm{d} s \\
& \left.-\int_{0}^{1} I^{\alpha, \rho}\left(s, u(s),{ }^{C} D_{0^{+}}^{\alpha-1, \rho} u(s),{ }^{C} D_{0^{+}}^{\alpha-2, \rho} u(s),{ }^{C} D_{0^{+}}^{\alpha-3, \rho} u(s)\right) \mathrm{d} s\right] .
\end{aligned}
$$

Set

$$
\begin{aligned}
\sup _{t \in[0,1]}|f(t, 0,0,0,0)| & =f_{0}, \\
r & \geq \frac{f_{0} A}{1-L A} .
\end{aligned}
$$

We shall show that $\mathscr{L} B_{r} \subset B_{r}$, where $B_{r}=\{u \in C([0,1], R):\|u\| \leq r\}$. For $u \in B_{r}$, we have

$$
\begin{aligned}
|\mathscr{L} u(t)| \leq & I^{\alpha, \rho}\left|f\left(s, u(s),{ }^{C} D_{0^{+}}^{\alpha-1, \rho} u(s),{ }^{C} D_{0^{+}}^{\alpha-2, \rho} u(s),{ }^{C} D_{0^{+}}^{\alpha-3, \rho} u(s)\right)\right| \\
& +\frac{\left|t^{3 \rho}\right|}{\rho^{3}|\psi|}\left[\sum_{i=1}^{m} \delta_{i} I^{\alpha+\beta, \rho}\left|f\left(s, u(s),{ }^{C} D_{0^{+}}^{\alpha-1, \rho} u(s),{ }^{C} D_{0^{+}}^{\alpha-2, \rho} u(s),{ }^{C} D_{0^{+}}^{\alpha-3, \rho} u(s)\right)\right|\right. \\
& \left.-\int_{0}^{1} I^{\alpha, \rho}\left|f\left(s, u(s),{ }^{C} D_{0^{+}}^{\alpha-1, \rho} u(s),{ }^{C} D_{0^{+}}^{\alpha-2, \rho} u(s),{ }^{C} D_{0^{+}}^{\alpha-3, \rho} u(s)\right)\right| \mathrm{d} s\right] \\
\leq & I^{\alpha, \rho}\left[\left|f\left(s, u(s),{ }^{C} D_{0^{+}}^{\alpha-1, \rho} u(s),{ }^{C} D_{0^{+}}^{\alpha-2, \rho} u(s),{ }^{C} D_{0^{+}}^{\alpha-3, \rho} u(s)\right)-f(s, 0,0,0,0)\right|\right.
\end{aligned}
$$




$$
\begin{aligned}
& +[|f(s, 0,0,0,0)|]+\frac{t^{3 \rho}}{\rho^{3}|\psi|}\left\{\sum_{i=1}^{m} \delta_{i} I^{\alpha+\beta, \rho}\right. \\
& \cdot\left[\left|f\left(s, u(s),{ }^{C} D_{0^{+}}^{\alpha-1, \rho} u(s),{ }^{C} D_{0^{+}}^{\alpha-2, \rho} u(s),{ }^{C} D_{0^{+}}^{\alpha-3, \rho} u(s)\right)-f(s, 0,0,0,0)\right|\right. \\
& +[|f(s, 0,0,0,0)|] \\
& -\int_{0}^{1} I^{\alpha, \rho}\left[\left|f\left(s, u(s),{ }^{C} D_{0^{+}}^{\alpha-1, \rho} u(s),{ }^{C} D_{0^{+}}^{\alpha-2, \rho} u(s),{ }^{C} D_{0^{+}}^{\alpha-3, \rho} u(s)\right)-f(s, 0,0,0,0)\right|\right. \\
& +\{[|f(s, 0,0,0,0)|] \mathrm{d} s\} .
\end{aligned}
$$

Therefore,

$$
\begin{aligned}
|\mathscr{L} u(t)| \leq & \sup _{t \in[0,1]}\left[L\left(|u(t)|+\left|{ }^{C} D_{0^{+}}^{\alpha-1, \rho} u(t)\right|+\left|{ }^{C} D_{0^{+}}^{\alpha-2, \rho} u(t)\right|+\left|{ }^{C} D_{0^{+}}^{\alpha-3, \rho} u(t)\right|\right)+f_{0}\right] \\
& \cdot\left[\frac{t^{\alpha \rho}}{\rho^{\alpha} \Gamma(\alpha+1)}+\frac{t^{3 \rho}}{\rho^{3}|\psi|}\left(\sum_{i=1}^{m} \delta_{i} \frac{\xi_{i}^{\rho(\alpha+\beta)}}{\rho^{\alpha+\beta} \Gamma(\alpha+\beta+1)}-\frac{1}{\rho^{\alpha} \Gamma(\alpha+1)(\rho \alpha+1)}\right)\right] \\
\leq & \left(L\|u\|+f_{0}\right)\left[\frac{1}{\rho^{\alpha} \Gamma(\alpha+1)}+\frac{1}{\rho^{3}|\psi|}\left(\sum_{i=1}^{m} \delta_{i} \frac{\xi_{i}^{\rho(\alpha+\beta)}}{\rho^{\alpha+\beta} \Gamma(\alpha+\beta+1)}\right.\right. \\
& \left.\left.\quad \frac{1}{\rho^{\alpha} \Gamma(\alpha+1)(\rho \alpha+1)}\right)\right] \\
\leq & \left(L\|u\|+f_{0}\right) A \leq\left(L r+f_{0}\right) A \leq r .
\end{aligned}
$$

Similarly,

$$
\begin{aligned}
& \left|\left({ }^{C} D_{0^{+}}^{\alpha-1, \rho} \mathscr{L} u\right)(t)\right| \leq\left(L\|u\|+f_{0}\right) A \leq\left(L r+f_{0}\right) A \leq r, \\
& \left|\left({ }^{C} D_{0^{+}}^{\alpha-2, \rho} \mathscr{L} u\right)(t)\right| \leq\left(L\|u\|+f_{0}\right) A \leq\left(L r+f_{0}\right) A \leq r, \\
& \left|\left({ }^{C} D_{0^{+}}^{\alpha-3, \rho} \mathscr{L} u\right)(t)\right| \leq\left(L\|u\|+f_{0}\right) A \leq\left(L r+f_{0}\right) A \leq r,
\end{aligned}
$$

By taking the norm from $t \in[0,1]$, it yields $\|\mathscr{L} u\| \leq r,\left\|^{C} D_{0^{+}}^{\alpha-1, \rho} \mathscr{L} u\right\| \leq r,\left\|^{C} D_{0^{+}}^{\alpha-2, \rho} \mathscr{L} u\right\| \leq r, \quad$ and $\left\|{ }^{C} D_{0^{+}}^{\alpha-3, \rho} \mathscr{L} u\right\| \leq r$. Consequently, we have

$$
\|\mathscr{L} u\|_{X} \leq\left(\operatorname{Lr}+f_{0}\right) A \leq r,
$$

which shows that $\mathscr{L}$ maps $B_{r}$ into itself. In order to show that the operator $\mathscr{L}$ is a contraction, let $u, v \in C([0,1], \mathbb{R})$. Then, for each $t \in[0,1]$, we obtain

$$
\begin{aligned}
\|\mathscr{L} u-\mathscr{L} v\|= & \sup _{t \in[0,1]}|\mathscr{L} u(t)-\mathscr{L} v(t)| \\
= & \sup _{t \in[0,1]} \mid I^{\alpha, \rho} f\left(s, u(s),{ }^{C} D_{0^{+}}^{\alpha-1, \rho} u(s),{ }^{C} D_{0^{+}}^{\alpha-2, \rho} u(s),{ }^{C} D_{0^{+}}^{\alpha-3, \rho} u(s)\right) \\
& +\frac{t^{3 \rho}}{\rho^{3}|\psi|}\left[\sum_{i=1}^{m} \delta_{i} I^{\alpha+\beta, \rho} f\left(s, u(s),{ }^{C} D_{0^{+}}^{\alpha-1, \rho} u(s),{ }^{C} D_{0^{+}}^{\alpha-2, \rho} u(s),{ }^{C} D_{0^{+}}^{\alpha-3, \rho} u(s)\right)\right. \\
& \left.-\int_{0}^{1} I^{\alpha, \rho} f\left(s, u(s),{ }^{C} D_{0^{+}}^{\alpha-1, \rho} u(s),{ }^{C} D_{0^{+}}^{\alpha-2, \rho} u(s),{ }^{C} D_{0^{+}}^{\alpha-3, \rho} u(s)\right) \mathrm{d} s\right]
\end{aligned}
$$




$$
\begin{aligned}
& -I^{\alpha, \rho} f\left(s, v(s),{ }^{C} D_{0^{+}}^{\alpha-1, \rho} v(s),{ }^{C} D_{0^{+}}^{\alpha-2, \rho} v(s),{ }^{C} D_{0^{+}}^{\alpha-3, \rho} v(s)\right) \\
& -\frac{t^{3 \rho}}{\rho^{3}|\psi|}\left[\sum_{i=1}^{m} \delta_{i} I^{\alpha+\beta, \rho} f\left(s, v(s),{ }^{C} D_{0^{+}}^{\alpha-1, \rho} v(s),{ }^{C} D_{0^{+}}^{\alpha-2, \rho} v(s),{ }^{C} D_{0^{+}}^{\alpha-3, \rho} v(s)\right)\right. \\
& \left.+\int_{0}^{1} I^{\alpha, \rho} f\left(s, v(s),{ }^{C} D_{0^{+}}^{\alpha-1, \rho} v(s),{ }^{C} D_{0^{+}}^{\alpha-2, \rho} v(s),{ }^{C} D_{0^{+}}^{\alpha-3, \rho} v(s)\right) d s\right] \mid \\
\leq & \sup _{t \in[0,1]}\left[L \left(|u(t)-v(t)|+\left|{ }^{C} D_{0^{+}}^{\alpha-1, \rho} u(t)-{ }^{C} D_{0^{+}}^{\alpha-1, \rho} v(t)\right|\right.\right. \\
& \left.\left.+\left|{ }^{C} D_{0^{+}}^{\alpha-2, \rho} u(t)-{ }^{C} D_{0^{+}}^{\alpha-2, \rho} v(t)\right|+\left|{ }^{C} D_{0^{+}}^{\alpha-3, \rho} u(t)-{ }^{C} D_{0^{+}}^{\alpha-3, \rho} v(t)\right|\right)\right] \\
& \cdot\left[\frac{1}{\rho^{\alpha} \Gamma(\alpha+1)}+\frac{1}{\rho^{3}|\psi|}\left(\sum_{i=1}^{m} \delta_{i} \frac{\xi_{i}^{\rho(\alpha+\beta)}}{\rho^{\alpha+\beta} \Gamma(\alpha+\beta+1)}-\frac{1}{\rho^{\alpha} \Gamma(\alpha+1)(\rho \alpha+1)}\right)\right] \\
\leq & L\|u-v\|\left[\frac{1}{\rho^{\alpha} \Gamma(\alpha+1)}+\frac{1}{\rho^{3}|\psi|}\left(\sum_{i=1}^{m} \delta_{i} \frac{\xi_{i}^{\rho(\alpha+\beta)}}{\rho^{\alpha+\beta} \Gamma(\alpha+\beta+1)}\right.\right. \\
\leq & L A\|u-v\| . \\
& \left.\left.-\frac{1}{\rho^{\alpha} \Gamma(\alpha+1)(\rho \alpha+1)}\right)\right]
\end{aligned}
$$

Similarly,

$$
\begin{aligned}
& \left\|\left({ }^{C} D_{0^{+}}^{\alpha-1, \rho} \mathscr{L} u\right)-\left({ }^{C} D_{0^{+}}^{\alpha-1, \rho} \mathscr{L} v\right)\right\| \leq L A\|u-v\|, \\
& \left\|\left({ }^{C} D_{0^{+}}^{\alpha-2, \rho} \mathscr{L} u\right)-\left({ }^{C} D_{0^{+}}^{\alpha-2, \rho} \mathscr{L} v\right)\right\| \leq L A\|u-v\|, \\
& \left\|\left({ }^{C} D_{0^{+}}^{\alpha-3, \rho} \mathscr{L} u\right)-\left({ }^{C} D_{0^{+}}^{\alpha-3, \rho} \mathscr{L} v\right)\right\| \leq L A\|u-v\| .
\end{aligned}
$$

Consequently,

$$
\|(\mathscr{L} u)-(\mathscr{L} v)\|_{X} \leq L A\|u-v\|_{X} .
$$

Thus, in view of condition (59), it follows that the operator $\mathscr{L}$ is a contraction. Hence, the operator $\mathscr{L}$ has a unique fixed point which corresponds to a unique solution of GFBVP (1).

We conclude this section with some examples showing the applicability of our main results.

Example 1. Let us consider the following boundary value problem:

$$
\left\{\begin{array}{l}
{ }^{C} D_{0^{+}}^{(7 / 2),(1 / 3)} u(t)=f\left(t, u(t),{ }^{C} D_{0^{+}}^{\alpha-1, \rho} u(t),{ }^{C} D_{0^{+}}^{\alpha-2, \rho} u(t),{ }^{C} D_{0^{+}}^{\alpha-3, \rho} u(t)\right), \quad t \in[0,1] \\
u(0)=\delta u(0)=\delta^{2} u(0)=0, \\
\int_{0}^{1} u(s) \mathrm{d} s=\sum_{i=1}^{2} \delta_{i} I^{(1 / 2),(1 / 3)} u\left(\xi_{i}\right),
\end{array}\right.
$$

where $\alpha=7 / 2, \beta=1 / 2, \rho=1 / 3, \delta_{1}=2 / 3, \delta_{2}=7 / 6, \xi_{1}=1 / 3$,

$\xi_{2}=5 / 6$, and

$$
\begin{aligned}
f\left(t, u(t),{ }^{C} D_{0^{+}}^{(5 / 2),(1 / 3)} u(t),{ }^{C} D_{0^{+}}^{(3 / 2),(1 / 3)} u(t),{ }^{C} D_{0^{+}}^{(1 / 2),(1 / 3)} u(t)\right)= & \frac{1}{9} e^{t} \sin t+\frac{u(t)}{(t+3)^{2}} \\
& +\frac{\left|{ }^{C} D_{0^{+}}^{(5 / 2),(1 / 3)} u(t)\right|}{t^{2}+20}+\frac{\left|{ }^{C} D_{0^{+}}^{(3 / 2),(1 / 3)} u(t)\right|}{5 \sqrt{10 t+64}+\frac{\left|{ }^{C} D_{0^{+}}^{(1 / 2),(1 / 3)} u(t)\right|}{11(t+1)}} .
\end{aligned}
$$


To check that the condition $\left(H_{2}\right)$ holds, let $u, v \in C([0,1], \mathbb{R})$ and $t \in[0,1]$; then, we get

$$
\begin{aligned}
\mid f(t, & \left.u(t),{ }^{C} D_{0^{+}}^{(5 / 2),(1 / 3)} u(t),{ }^{C} D_{0^{+}}^{(3 / 2),(1 / 3)} u(t),{ }^{C} D_{0^{+}}^{(1 / 2),(1 / 3)} u(t)\right) \\
& -f\left(t, v(t),{ }^{C} D_{0^{+}}^{(5 / 2),(1 / 3)} v(t),{ }^{C} D_{0^{+}}^{(3 / 2),(1 / 3)} v(t),{ }^{C} D_{0^{+}}^{(1 / 2),(1 / 3)} v(t)\right) \mid \\
\leq & \frac{1}{9}|u(t)-v(t)|+\frac{1}{20}\left|{ }^{C} D_{0^{+}}^{(5 / 2),(1 / 3)} u(t)-{ }^{C} D_{0^{+}}^{(5 / 2),(1 / 3)} v(t)\right|+\frac{1}{40}\left|{ }^{C} D_{0^{+}}^{(3 / 2),(1 / 3)} u(t)-{ }^{C} D_{0^{+}}^{(3 / 2),(1 / 3)} v(t)\right| \\
& +\frac{1}{11}\left|{ }^{C} D_{0^{+}}^{(1 / 2),(1 / 3)} u(t)-{ }^{C} D_{0^{+}}^{(1 / 2),(1 / 3)} v(t)\right| \leq \frac{1}{9}\|u-v\|_{X} .
\end{aligned}
$$

Hence, the condition $\left(H_{2}\right)$ holds with $L=1 / 9$ and it follows that $L A=0.497230703<1$, where $A=\sigma+K V$ and $V, K$ and $\sigma$ are given by (43)-(45). Then, the hypotheses of Theorem 7 are satisfied and problem (69) has a unique solution on $[0,1]$.
Example 2. We consider the following boundary value problem:

$$
\left\{\begin{array}{l}
{ }^{C} D_{0^{+}}^{(7 / 2),(1 / 3)} u(t)=f\left(t, u(t),{ }^{C} D_{0^{+}}^{\alpha-1, \rho} u(t),{ }^{C} D_{0^{+}}^{\alpha-2, \rho} u(t),{ }^{C} D_{0^{+}}^{\alpha-3, \rho} u(t)\right), \quad t \in[0,1] \\
u(0)=\delta u(0)=\delta^{2} u(0)=0, \\
\int_{0}^{1} u(s) \mathrm{d} s=\sum_{i=1}^{2} \delta_{i} I^{(1 / 2),(1 / 3)} u\left(\xi_{i}\right) .
\end{array}\right.
$$

where $\quad \alpha=7 / 2, \beta=1 / 2, \rho=1 / 3, \delta_{1}=2 / 3, \delta_{2}=7 / 6, \xi_{1}=$ $1 / 3$, and $\xi_{2}=5 / 6$. Choose

$$
\begin{aligned}
f(t, & \left.u(t),{ }^{C} D_{0^{+}}^{(5 / 2),(1 / 3)} u(t),{ }^{C} D_{0^{+}}^{(3 / 2),(1 / 3)} u(t),{ }^{C} D_{0^{+}}^{(1 / 2),(1 / 3)} u(t)\right) \\
= & \frac{1}{30} e^{t}+\frac{\tan ^{-1} u(t)}{\sqrt{\cos t+145}}+\frac{t}{8 t^{3}+30}\left(\frac{\left|{ }^{C} D_{0^{+}}^{(5 / 2),(1 / 3)} u(t)\right|}{\left|{ }^{C} D_{0^{+}}^{(5 / 2),(1 / 3)} u(t)\right|+1}\right) \\
& +\frac{3}{6(t+10)}\left(\frac{\left|{ }^{C} D_{0^{+}}^{(3 / 2),(1 / 3)} u(t)\right|}{\left|{ }^{C} D_{0^{+}}^{(3 / 2),(1 / 3)} u(t)\right|+1}\right)+\frac{1}{\sqrt{\sin t+169}}\left(\frac{\left|{ }^{C} D_{0^{+}}^{(1 / 2),(1 / 3)} u(t)\right|}{\left|{ }^{C} D_{0^{+}}^{(1 / 2),(1 / 3)} u(t)\right|+1}\right) .
\end{aligned}
$$

Set $F(u)=|u|^{\alpha} / \alpha, \alpha>1$. In particular, if $\alpha=2$, then $F$ is an $\mathrm{N}$-function and satisfies

$$
\int_{0}^{1} F(|f(u(s))|) \mathrm{d} s<\infty
$$

which shows that $f$ belongs to an Orlicz space $L_{F}([0,1])$. Furthermore,

$$
\begin{aligned}
& \left|f\left(t, u(t),{ }^{C} D_{0^{+}}^{(5 / 2),(1 / 3)} u(t),{ }^{C} D_{0^{+}}^{(3 / 2),(1 / 3)} u(t),{ }^{C} D_{0^{+}}^{(1 / 2),(1 / 3)} u(t)\right)\right| \\
& \quad \leq \mathscr{M}(t),
\end{aligned}
$$

with $\mathscr{M}(t)=\left(e^{t} / 30\right)+(\pi / 2 \sqrt{\cos t+145})+\left(t /\left(8 t^{3}+30\right)\right)+$ $(3 / 6(t+10))+(1 / \sqrt{\sin t+169})$ and $\|\mathscr{M}\|=1.225$. Thus, the condition $\left(H_{1}\right)$ holds and by the conclusion of Theorem 5 , problem $(72)$ has at least one solution on $[0,1]$. 
Moreover,

$$
\begin{aligned}
\mid f(t, & \left.u(t),{ }^{C} D_{0^{+}}^{(5 / 2),(1 / 3)} u(t),{ }^{C} D_{0^{+}}^{(3 / 2),(1 / 3)} u(t),{ }^{C} D_{0^{+}}^{(1 / 2),(1 / 3)} u(t)\right) \\
& -f\left(t, v(t),{ }^{C} D_{0^{+}}^{(5 / 2),(1 / 3)} v(t),{ }^{C} D_{0^{+}}^{(3 / 2),(1 / 3)} v(t),{ }^{C} D_{0^{+}}^{(1 / 2),(1 / 3)} v(t)\right) \mid \\
\leq & \frac{1}{12}|u(t)-v(t)|+\frac{1}{30}\left|{ }^{C} D_{0^{+}}^{(5 / 2),(1 / 3)} u(t)-{ }^{C} D_{0^{+}}^{(5 / 2),(1 / 3)} v(t)\right|+\frac{1}{20}\left|{ }^{C} D_{0^{+}}^{(3 / 2),(1 / 3)} u(t)-{ }^{C} D_{0^{+}}^{(3 / 2),(1 / 3)} v(t)\right| \\
& +\frac{1}{13}\left|{ }^{C} D_{0^{+}}^{(1 / 2),(1 / 3)} u(t)-{ }^{C} D_{0^{+}}^{(1 / 2),(1 / 3)} v(t)\right| \leq \frac{1}{12}\|u-v\|_{X} .
\end{aligned}
$$

which proves the validity of condition $\left(\mathrm{H}_{2}\right)$ with $L A=0.372923027<1$, where $A=\sigma+K V$ and $V, K$, and $\sigma$ are given by (43)-(45). Hence, Theorem 7 can be applicable and so problem (72) has a unique solution on $[0,1]$.

\section{Ulam Stability}

In this section, we develop the criteria for Ulam stability of GFBVP (1) by means of its equivalent integral equation. For simplicity, we set

$$
f\left(s, v(s),{ }^{C} D_{0^{+}}^{\alpha-1, \rho} v(s),{ }^{C} D_{0^{+}}^{\alpha-2, \rho} v(s),{ }^{C} D_{0^{+}}^{\alpha-3, \rho} v(s)\right)=\widehat{f(s)} .
$$

Then,

$$
\begin{aligned}
v(t)= & \frac{\rho^{1-\alpha}}{\Gamma(\alpha)} \int_{0}^{t}\left(t^{\rho}-s^{\rho}\right)^{\alpha-1} s^{\rho-1} \widehat{f(s)} \mathrm{d} s \\
& +\frac{t^{3 \rho}}{\rho^{3} \psi}\left[\sum_{i=1}^{m} \delta_{i} \frac{\rho^{1-(\alpha+\beta)}}{\Gamma(\alpha+\beta)} \int_{0}^{\xi_{i}}\left(\xi_{i}^{\rho}-s^{\rho}\right)^{\alpha+\beta-1} s^{\rho-1}\right. \\
& \left.\cdot \widehat{f(s)} \mathrm{d} s-\int_{0}^{1} I^{\alpha, \rho} \widehat{f(s)} \mathrm{d} s\right]
\end{aligned}
$$

where $v \in X$ and $f:[0,1] \times \mathbb{R}^{4} \longrightarrow \mathbb{R}$ is a continuous function. Next, we define a continuous nonlinear operator $\mathscr{F}: X \longrightarrow X$ as

$$
\mathscr{F} v(t)={ }^{C} D_{0^{+}}^{\alpha, \rho} v(t)-\widehat{f(t)} .
$$

For definitions of Ulam-Hyers, generalized Ulam-Hyers and Ulam-Hyers-Rassias stability, and we refer to [41].

Definition 7. GFBVP (1) is said to be Ulam-Hyers stable if there exists a real number $b>0$ such that, for each $\epsilon>0$ and for each solution $v \in X$,

$$
\|\mathscr{F} v\| \leq \epsilon, \quad t \in[0,1],
$$

there exists a solution $u \in X$ of (1) satisfying the inequality

$$
\|u-v\| \leq b \epsilon_{1}, \quad t \in[0,1]
$$

where $\epsilon_{1}$ is a positive real number depending on $\epsilon$.
Definition 8. GFBVP (1) is generalized Ulam-Hyers stable if there exists $\Theta \in C\left(\mathbb{R}^{+}, \mathbb{R}^{+}\right)$such that, for each solution $v \in X$ of (1), there exists a solution $u \in X$ of (1) with

$$
|u(t)-v(t)| \leq \Theta(\epsilon), \quad t \in[0,1] .
$$

Definition 9. GFBVP (1) is Ulam-Hyers-Rassias stable with respect to $\Psi \in C([0,1], \mathbb{R})$ if there exists a real number $b>0$ such that, for each $\epsilon>0$ and for each solution $v \in X$ of (1),

$$
|\mathscr{F} v(t)| \leq \epsilon \Psi(t), \quad t \in[0,1],
$$

we can find a solution $u \in X$ of (1) satisfying the inequality

$$
|u(t)-v(t)| \leq b \epsilon_{1} \Psi(t), \quad t \in[0,1],
$$

where $\epsilon_{1}$ is a positive real number depending on $\epsilon$.

Theorem 8. Assume that conditions (47) and (59) hold. Then, GFBVP (1) satisfies both Ulam-Hyers and generalized Ulam-Hyers stability criteria.

Proof. We know that $u \in X$ is a unique solution of (1) (by Theorem 7). Let $v \in X$ be another solution of (1) satisfying $\|\mathscr{F} v\| \leq \epsilon, t \in[0,1]$. Observe that the operators $\mathscr{F}$ and $\mathscr{L}-$ $\mathscr{I}$ are equivalent for every solution $v \in X$ of (1). Therefore, by the fixed point property of the operator $\mathscr{L}$ together with $u(t)=\mathscr{L} u(t)$ and $\|\mathscr{F} v\| \leq \epsilon, t \in[0,1]$, we have

$$
\begin{aligned}
|v(t)-u(t)| & =|v(t)-\mathscr{L} v(t)+\mathscr{L} v(t)-\mathscr{L} u(t)| \\
& \leq|\mathscr{L} u(t)-\mathscr{L} v(t)|+|\mathscr{L} v(t)-v(t)| \\
& \leq L A\|u-v\|_{X}+\epsilon,
\end{aligned}
$$

where $\epsilon>0$ and $L A<1$. Taking the norm for $t \in[0,1]$ and solving for $\|u-v\|_{X}$, we obtain

$$
\|u-v\|_{X} \leq \frac{\epsilon}{1-L A}
$$

Choosing $\epsilon_{1}=\epsilon /(1-L A)$ and $b=1$, the Ulam-Hyers stability condition is satisfied. More generally, defining $\Theta(\epsilon)=\epsilon /(1-L A)$, the generalized Ulam-Hyers stability condition is also satisfied which completes the proof.

Theorem 9. Assume that conditions (47) and (59) hold and there exists a function $z \in C([0,1], \mathbb{R})$ satisfying condition 
$\|u(t)-v(t)\| \leq b \epsilon_{1} \Psi(t), t \in[0,1]$. Then, GFBVP (1) is Ulam-Hyers-Rassias stable with respect to $\Psi$.

Proof. Similar to the discussion in the proof of Theorem 8, we have

$$
\|u-v\|_{X} \leq \epsilon_{1} \Psi(t),
$$

with $\epsilon_{1}=\epsilon /(1-L A)$, and we get the desired result.

\section{Data Availability}

No data were used to support this study.

\section{Conflicts of Interest}

The authors declare no conflicts of interest.

\section{Authors' Contributions}

Wafa Shammakh, Hadeel Z. Alzumi, and Zahra Albarqi contributed to the design and implementation of the research, to the analysis of the results, and to the writing of the manuscript.

\section{References}

[1] R. R. Nigmatullin, T. Omay, and D. Baleanu, "On fractional filtering versus conventional filtering in economics," Communications in Nonlinear Science and Numerical Simulation, vol. 15, no. 4, pp. 979-986, 2010.

[2] M. P. Lazarević, "Finite time stability analysis of $P D^{\alpha}$ fractional control of robotic time-delay systems," $M e-$ chanics Research Communications, vol. 33, pp. 269-279, 2006.

[3] K. W. Blayneh, "Analysis of age structured host-parasitoid model," Far East Journal of Dynamical Systems, vol. 4, pp. 125-145, 2002.

[4] A. A. Kilbas, H. M. Srivastava, and J. J. Trujillo, Theory andApplications of Fractional Differential Equations, vol. 204, Elsevier, Amsterdem, Netherlands, 2006.

[5] Z. Liu and J. Sun, "Nonlinear boundary value problems of fractional differential systems," Computers \& Mathematics with Applications, vol. 64, no. 4, pp. 463-475, 2012.

[6] X. Meng, Z. Li, and J. J. Nieto, "Dynamic analysis of Michaelis-Menten chemostat-type competition models with time delay and pulse in a polluted environment," Journal of Mathematical Chemistry, vol. 47, no. 1, pp. 123-144, 2010.

[7] K. S. Miller and B. Ross, An Introduction to the Fractional Calculus and Fractional Differential Equations, John Wiley, New York, USA, 1993.

[8] I. Podlubny, Fractional Differential Equations, Vol. 198, Academic Press, San Diego, USA, 1999.

[9] F. A. Rihan, Q. M. Al-Mdallal, H. J. AlSakaji, and A. Hashish, "A fractional-order epidemic model with time-delay and nonlinear incidence rate," Chaos, Solitons \& Fractals, vol. 126, pp. 97-105, 2019.

[10] T. Abdeljawad, Q. M. Al-Mdallal, and F. Jarad, "Fractional logistic models in the frame of fractional operators generated by conformable derivatives," Chaos, Solitons \& Fractals, vol. 119, pp. 94-101, 2019.

[11] J. Jiang and H. Wang, "Existence and uniqueness of solutions for a fractional differential equation with multi-point boundary value problems," Journal of Applied Analysis \& Computation, vol. 9, no. 6, pp. 2156-2168, 2019.
[12] p. Chen and Y. Gao, "Positive solutions for a class of nonlinear fractional differential equations with nonlocal boundary value conditions," Positivity, vol. 22, no. 3, pp. 761-772, 2018.

[13] Q. Leng, J. Yin, and p. Yan, "Existence and uniqueness of positive solutions of fractional differential equations with infinite-point boundary value conditions," Journal of Computational Analysis and Applications, vol. 26, no. 2, pp. 280-287, 2019.

[14] H. Zhai, C. Yu, and J. Wang, "Existence of solutions for a class of fractional ordinary differential equations with integral and antiperiodic boundary value conditions," in Proceedings of the Chinese Control And Decision Conference (CCDC), pp. 883888, Shenyang, China, June 2018.

[15] Q. Sun, H. Ji, and Y. Cui, "Positive solutions for boundary value problems of fractional differential equation with integral boundary conditions," Journal of Function Spaces, vol. 2018, Article ID 6461930, 8 pages, 2018.

[16] U. N. Katugampola, "New approach to a generalized fractional integral," Applied Mathematics and Computation, vol. 218, no. 3, pp. 860-865, 2011.

[17] M. Caputo and M. Fabrizio, "A new definition of fractional derivative without singular kernal," Progress in Fractional Differentiation and Applications, vol. 1, no. 2, pp. 73-85, 2015.

[18] T. Abdeljawad and D. Baleanu, "Discrete fractional differences with nonsingular discrete Mittag-Leffler kernels," Advances in Difference Equations, vol. 2016, no. 1, p. 18, 2016.

[19] T. Abdeljawad, "Fractional difference operators with discrete generalized Mittag-Leffler kernels," Chaos, Solitons \& Fractals, vol. 126, pp. 315-324, 2019.

[20] F. Jarad, T. Abdeljawad, and J. Alzabut, "Generalized fractional derivatives generated by a class of local proportional derivatives," The European Physical Journal Special Topics, vol. 226, no. 16-18, pp. 3457-3471, 2017.

[21] A. Atangana and D. Baleanu, "New fractional derivatives with nonlocal and non-singular kernel: theory and application to heat transfer model," The Journal Thermal Science, vol. 20, no. 2, p. 8, 2016.

[22] N. Sene and J. F. G. Aguilar, "Fractional mass-spring-damper system described by generalized fractional order derivatives," Fractal and Fractional, vol. 3, no. 39, p. 15, 2019.

[23] F. Jarad and T. Abdeljawad, "Generalized fractional derivatives and Laplace transform," Discrete \& Continuous Dynamical Systems-S, vol. 13, no. 3, pp. 709-722, 2020.

[24] A. Alsaedi, M. Alghanmi, B. Ahmad, and S. K. Ntouyas, "Generalized Liouville-Caputo fractional differential equations and inclusions with nonlocal generalized fractional integral and multipoint boundary conditions," Symmetry, vol. 10, p. 21, 2018.

[25] W. Shammakh and H. Z. Alzumi, "Existence results for nonlinear fractional boundary value problem involving generalized proportional derivative," Advances in Difference Equations, vol. 2019, no. 94, p. 20, 2019.

[26] Y. Y. Gambo, R. Ameen, F. Jarad, and T. Abdeljawad, "Existence and uniqueness of solutions to fractional differential equations in the frame of generalized Caputo fractional derivatives," Advances in Difference Equations, vol. 2018, no. 134, p. 14, 2018.

[27] R. Almeide, A. B. Malinowska, and T. Odzijewicz, "Fractional differential equations with dependence on the CaputoKatugampola derivative," Journal of Computational and Nonlinear Dynamics, vol. 11, no. 6, p. 19, 2016.

[28] A. Khan, M. I. Syam, A. Zada, and H. Khan, "Stability analysis of nonlinear fractional differential equations with Caputo and 
Riemann-Liouville derivatives," The European Physical Journal Plus, vol. 133, no. 264, p. 9, 2018.

[29] A. Khan, H. Khan, J. F. Gómez-Aguilar, and T. Abdeljawad, "Existence and Hyers-Ulam stability for a nonlinear singular fractional differential equations with Mittag-Leffler kernel," Chaos, Solitons \& Fractals, vol. 127, pp. 422-427, 2019.

[30] H. Khan, T. Abdeljawad, M. Aslam, R. A. Khan, and A. Khan, "Existence of positive solution and Hyers-Ulam stability for a nonlinear singular-delay-fractional differential equation," Advances in Difference Equations, vol. 2019, no. 104, p. 13, 2019.

[31] Z. Birnbaum and W. Orlicz, "Über die Verallgemeinerung des Begriffes der zueinander konjugierten Potenzen," Studia Mathematica, vol. 3, p. 67, 1931.

[32] R. Ayazoglu, M. Avci, and N. T. Chung, "Existence of solutions for nonlocal problems in Orlicz-Sobolev spaces via monotone method," Electronic Journal of Mathematical Analysis and Applications, vol. 4, no. 1, pp. 63-73, 2016.

[33] S. Bahrouni, H. Ounaies, and L. S. Tavares, "Basic results of fractional Orlicz-Sobolev space and applications to non-local problems," 2019, https://arxiv.org/abs/1901.00784.

[34] U. N. Katugampola, "Existence and uniqueness results for a class of generalized fractional differential equation," Bulletin of Mathematical Analysis and Applications, vol. 6, no. 4, p. 15, 2014.

[35] U. N. Katugampola, "A new approach to generalized fractional derivatives," Bulletin of Mathematical Analysis and Applications, vol. 6, no. 4, p. 15, 2014.

[36] M. A. Krasnosel'skii and Y. B. Rutickii, Convex Functions and Orlicz Spaces, P. Noordhoff Ltd., Groningen, Netherlands, 1961.

[37] J. Musielak, Orlicz Spaces and Modular Spaces, Vol. 1034, Springer, New York, USA, 2006.

[38] F. Jarad, T. Abdeljawad, and D. Baleanu, "On the generalized fractional derivatives and their Caputo modification," The Journal of Nonlinear Sciences and Applications, vol. 10, no. 5, pp. 2607-2619, 2017.

[39] Y. Y. Gambo, F. Jarad, D. Baleanu, and T. Abdeljawad, "Fractional vector calculus in the frame of a generalized Caputo fractional derivative," UPB Scientific Bulletin, Series A: Applied Mathematics and Physics, vol. 80, no. 4, pp. 219-228, 2018.

[40] D. R. Smart, Fixed Point Theorems, Cambridge University Press, Cambridge, UK, 1980.

[41] S.-M. Jung, Hyers-Ulam-Rassias Stability of Functional Equations in Nonlinear Analysis, Springer, New York, USA, 2011. 